\title{
Asian rainbow option pricing formulas of uncertain stock model
}

\author{
Rong Gao ${ }^{1} \cdot$ Wei $\mathrm{Wu}^{1} \cdot$ Jie $\mathrm{Liu}^{2}$ \\ Accepted: 28 May 2021 / Published online: 7 June 2021 \\ (c) The Author(s), under exclusive licence to Springer-Verlag GmbH Germany, part of Springer Nature 2021
}

\begin{abstract}
Asian rainbow option is option on the minimum or the maximum of several average prices. In modern financial market, Asian rainbow option is an effective instrument for asset allocation and risk management. The investor with Asian rainbow option enjoys an entitlement to select a max or min from multiple assets with an exercise price at maturity date. The investor has to defray fee to acquire this right, which raises the option pricing issue. This paper mainly explores the pricing of Asian rainbow option in the uncertain financial environment, in which the underlying assets prices are treated as uncertain processes. Here, the pricing formulas of Asian rainbow option are derived under the condition that stock prices obey uncertain differential equations driven by independent Liu processes. Furthermore, some numerical examples are designed to compute the prices of these options.
\end{abstract}

Keywords Option pricing $\cdot$ Asian option $\cdot$ Rainbow option $\cdot$ Uncertain differential equation

\section{Introduction}

In a complex financial market, option is often used as a tool to gain high returns at small costs. The owner of the option may wield or waive the right to trade an asset at a certain price on a specified date. The investor has to pay fee to obtain the option, which causes the issue of option pricing. Based on the stochastic differential equation, the famous BlackScholes formula was presented by Black and Scholes (1973), which was a major breakthrough in option pricing theory. Since then, the stock price was depicted by various stochastic differential equations. Merton (1976) put forward a jumpdiffusion model in which the continuous and jump processes determine the return of the underlying stock. Cox and Ross (1976) proposed the constant elasticity of variance model. The advancement of option pricing theory greatly promotes the derivation of option types.

The theoretical study of Asian option started with the work of Ingersoll (1987). The payoff of Asian option is determined

\footnotetext{
Rong Gao

rgao@hebut.edu.cn

hliujie@hgnu.edu.cn

1 School of Economics and Management, Hebei University of Technology, Tianjin 300401, China

2 College of Mathematics and Statistics, Huanggang Normal University, Huanggang 438000, China
}

$凶$ Jie Liu by the average price of underlying asset over its lifetime. Benefited from the averaging feature, Asian option can decrease the volatility of underlying asset price and the risk brought by market manipulation occurring near the expiry date. Sun and Chen (2015) contended that Asian option has a lower premium than standard option. In the Black-Scholes setting, Willems (2019) derived orthogonal polynomial expansion for the price of a continuous arithmetic Asian option. Under general stochastic asset model, Fusai and Kyriakou (2016) developed the pricing method of continuous and discrete arithmetic Asian options. For pricing discrete arithmetic Asian option, a transform-based algorithm was presented by Corsaro et al. (2019). By means of two finite difference methods, the pricing formulas of Asian option were deduced by Mudzimbabwe et al. (2012).

Asian rainbow option raised by $\mathrm{Wu}$ and Zhang (1999) is a mixture of Asian option and rainbow option, which is widely used in incentive contracts design and risk management. Due to the characteristic of rainbow option, Asian rainbow option is a type of multi-asset option. For the literature on multi-asset option pricing, interested readers can refer to references (Johnson 1987; Margrabe 1978; Ouwehand and West 2006; Stulz 1982). Thus, the payoff of Asian rainbow option is related to the averages of the prices of several underlying assets over certain time interval. Based on the arbitrage-free principle and the Ito lemma, Bin and Fei (2009) provided the pricing formula of geometric Asian rain- 
bow option with known dividends. Within a Black-Scholes environment, Zhan and Cheng (2010) came up with a simple method to price Asian rainbow option on dividend-paying assets. By using fractional Brownian motion to portray the underlying assets prices, Wang et al. (2018) studied the pricing problem of Asian rainbow option. Under the mixed fractional Brownian motion, Ahmadian and Ballestra (2020) deduced the closed-form solution of geometric Asian rainbow options.

As well known, the traditional option pricing model is established on the basis of probability theory, which regards underlying asset price as the Wiener process. As can be seen from the law of large numbers, the application of probability theory requires that the cumulative probability distribution approximates the real frequency very much. Therefore, it is essential to obtain enough samples. Nevertheless, it is sometimes difficult to acquire adequate samples for each indeterministic events. For example, during the coronavirus outbreak, we do not know the demand for masks in the harderhit areas. We need to invite the domain experts to evaluate the belief degree, which is largely determined by individual awareness and preferences for the indeterministic factors. For processing belief degrees mathematically, uncertainty theory was pioneered by Liu $(2009,2012)$. Uncertain variable is the basic tool in uncertainty theory, which was proposed to depict uncertain quantity. For describing the change of uncertain variable with time, Liu (2008) came up with the conception of uncertain process.

As a special uncertain process, Liu process provided by Liu (2009) can be considered as a counterpart of the Wiener process. For the differential equation driven by the Wiener process, its noise term is generally understood as a normal random variable which has expected value 0 and variance $\infty$. Then, the stock price has infinite changes at any moment, which goes against the reality. Hence, it is more reasonable to use Liu process to portray the stock price (Liu 2013). Driven by Liu process, uncertain differential equation was presented by Liu (2009). By employing uncertain differential equation, Liu (2009) proposed an uncertain stock model and discovered the European option pricing formula. Following uncertain stock model, the pricing formulas of Asian barrier option and American barrier option were deduced by Yang et al. (2019) and Gao et al. (2019), respectively. Lu et al. (2019) brought forward a new uncertain stock model and explored the pricing of European option. Tian et al. (2019) studied the pricing problem of European barrier option under the uncertain mean-reverting stock model.

In this paper, the average prices of underlying assets is calculated by the arithmetic mean and geometric mean methods. We regard the underlying assets prices as uncertain processes and provide the pricing formulas of Asian rainbow option based on the uncertain stock model. The structure of the paper is organized below. Section 2 will briefly state some related theorems and definitions of uncertainty theory. Section 3 will explore the pricing of arithmetic Asian put 2 and call 1 option, and geometric Asian put 2 and call 1 option. In Sect. 4, Asian rainbow call options will be investigated, including Asian rainbow call on max options and Asian rainbow call on min options. And their option pricing formulas are obtained by rigorous deductions. Four types of Asian rainbow put options will be analyzed in Sect. 5. Similar to Sect. 4 , the option pricing formulas in the above four cases are strictly deduced. Some brief conclusions are given in Sect. 6 .

\section{Preliminaries}

Definition 1 (Liu 2009, 2012) Providing that $(\Gamma, \mathcal{L})$ is a measurable space. A set function $\mathcal{M}: \mathcal{L} \rightarrow[0,1]$ is called an uncertain measure if it satisfies the following conditions, Axiom 1. (Normality Axiom) $\mathcal{M}\{\Gamma\}=1$ for the universal set $\Gamma$;

Axiom 2. (Duality Axiom) $\mathcal{M}\{\Lambda\}+\mathcal{M}\left\{\Lambda^{c}\right\}=1$ for any event $\Lambda$;

Axiom 3. (Subadditivity Axiom) For every countable sequence of events $\Lambda_{1}, \Lambda_{2}, \cdots$, we have

$\mathcal{M}\left\{\bigcup_{i=1}^{\infty} \Lambda_{i}\right\} \leq \sum_{i=1}^{\infty} \mathcal{M}\left\{\Lambda_{i}\right\}$

Axiom 4. (Product Axiom) Let $\left(\Gamma_{k}, \mathcal{L}_{k}, \mathcal{M}_{k}\right)(k=1,2, \cdots)$ represent uncertainty spaces. The product uncertain measure $\mathcal{N}$ is an uncertain measure satisfying

$\mathcal{M}\left\{\prod_{k=1}^{\infty} \Lambda_{k}\right\}=\bigwedge_{k=1}^{\infty} \mathcal{M}_{k}\left\{\Lambda_{k}\right\}$

where $\Lambda_{k}$ are arbitrary events chosen from $\mathcal{L}_{k}(k=$ $1,2, \cdots)$.

Theorem 1 (Liu 2010a, Monotonicity Theorem) For any events $\Lambda_{1}$ and $\Lambda_{2}$ with $\Lambda_{1} \subset \Lambda_{2}$, if the uncertain measure is a set function with monotone increasing feature, then we have

$\mathcal{M}\left\{\Lambda_{1}\right\} \leq \mathcal{M}\left\{\Lambda_{2}\right\}$

Theorem 2 (Liu 2013) A function $\Phi^{-1}(\alpha):(0,1) \rightarrow \Re$ is an inverse uncertainty distribution if and only if it is a continuous and strictly increasing function concerning $\alpha$.

Theorem 3 (Liu 2007) Assume that there is uncertainty distribution $\Phi$ for an uncertain variable $\xi$. If $E[\xi]$ exists, then

$E[\xi]=\int_{-\infty}^{+\infty} x \mathrm{~d} \Phi(x)$. 
Furthermore if $\Phi$ is regular, then we also have

$E[\xi]=\int_{0}^{1} \Phi^{-1}(\alpha) \mathrm{d} \alpha$.

Definition 2 (Liu 2009) An uncertain process $C_{t}$ is called a Liu process if

(i) $C_{0}=0$ and almost all sample paths are Lipschitz continuous,

(ii) $C_{t}$ has stationary and independent increments,

(iii) each increment $C_{s+t}-C_{s}$ is a normal uncertain variable owning expected value 0 and variance $t^{2}$, whose uncertainty distribution is

$$
\Phi(x)=\left(1+\exp \left(\frac{-\pi x}{\sqrt{3} t}\right)\right)^{-1}, x \in \Re
$$

Theorem 4 (Yao and Chen 2013) Suppose that there are the solution $X_{t}$ and $\alpha$-path $X_{t}^{\alpha}$ for an uncertain differential equation

$d X_{t}=f\left(t, X_{t}\right) d t+g\left(t, X_{t}\right) d C_{t}$

Then,

$$
\begin{aligned}
& \mathcal{N}\left\{X_{t} \leq X_{t}^{\alpha}, \forall t\right\}=\alpha, \\
& \mathcal{M}\left\{X_{t}>X_{t}^{\alpha}, \forall t\right\}=1-\alpha,
\end{aligned}
$$

where $X_{t}$ possesses an inverse uncertainty distribution

$\Psi_{t}^{-1}(\alpha)=X_{t}^{\alpha}$.

Theorem 5 (Yao 2013) Suppose that there are the solution $X_{t}$ and $\alpha$-path $X_{t}^{\alpha}$ for an uncertain differential equation

$d X_{t}=f\left(t, X_{t}\right) d t+g\left(t, X_{t}\right) d C_{t}$.

Then, the time integral $\int_{0}^{s} Y\left(X_{t}\right) d t$ possesses an inverse uncertainty distribution

$\Psi_{s}^{-1}(\alpha)=\int_{0}^{s} Y\left(X_{t}^{\alpha}\right) d t, s>0$

where $Y(x)$ is a function owning strictly increasing feature.

Theorem 6 (Liu 2010b) Let $\xi_{1}, \xi_{2}, \cdots, \xi_{n}$ be independent uncertain variables with regular uncertainty distributions $\Phi_{1}, \Phi_{2}, \cdots, \Phi_{n}$, respectively. If $f\left(\xi_{1}, \xi_{2}, \cdots, \xi_{n}\right)$ is strictly increasing with respect to $\xi_{1}, \xi_{2}, \cdots, \xi_{m}$ and strictly decreasing with respect to $\xi_{m+1}, \xi_{m+2}, \cdots, \xi_{n}$, then

$\xi=f\left(\xi_{1}, \xi_{2}, \cdots, \xi_{n}\right)$ has an inverse uncertainty distribution

$$
\begin{aligned}
& \Psi^{-1}(\alpha)=f\left(\Phi_{1}^{-1}(\alpha), \cdots, \Phi_{m}^{-1}(\alpha), \Phi_{m+1}^{-1}(1-\alpha),\right. \\
& \left.\cdots, \Phi_{n}^{-1}(1-\alpha)\right) .
\end{aligned}
$$

\section{Asian put 2 and call 1 option}

Asian put 2 and call 1 option provides the owner with an entitlement to exchange Asset 2 for Asset 1 on the expiration date. The gain of the option is related to the mean of two underlying assets prices at a predetermined time in the future. The means of underlying assets prices are calculated by the arithmetic average and geometric average methods. In this section, we study arithmetic Asian put 2 and call 1 option and geometric Asian put 2 and call 1 option. Moreover, the pricing formulas of corresponding option are obtained by strict deduction.

\subsection{Arithmetic Asian put 2 and call 1 option}

Arithmetic Asian put 2 and call 1 option is a kind of exchange option, in which market prices of Asset 1 and Asset 2 are calculated by arithmetic average method. The payoff of the option relies on the arithmetic mean of Asset 1 and Asset 2 market prices within an agreed period of time. The arithmetic mean of N numbers is defined as $\left(S_{1}+S_{2}+\cdots+S_{n}\right) / n$. When the underlying asset price $S_{t}$ changes continuously from time zero to time $T$, the arithmetic mean of $S_{t}$ can be written as $\int_{0}^{T} S_{t} d t / T$.

Considering that the underlying asset prices of arithmetic Asian put 2 and call 1 option with a maturity time $T$ obey different uncertain differential equations, the following model is proposed:

$$
\left\{\begin{array}{l}
d Y_{t}=\gamma Y_{t} d t \\
d S_{t}=\mu_{1} S_{t} d t+\sigma_{1} S_{t} d C_{1 t} \\
d X_{t}=\mu_{2} X_{t} d t+\sigma_{2} X_{t} d C_{2 t}
\end{array}\right.
$$

where $Y_{t}$ is the bond price, $S_{t}$ represents the price of Asset 1, $X_{t}$ is the price of Asset 2, $C_{1 t}$ and $C_{2 t}$ are independent Liu processes, $\sigma_{1}$ and $\sigma_{2}$ are, respectively, the log-diffusion of $S_{t}$ and $X_{t}, \mu_{1}$ and $\mu_{2}$ are, respectively, the log-drift of $S_{t}$ and $X_{t}$, and $\gamma$ is the riskless interest rate.

Since $C_{1 t}$ and $C_{2 t}$ are independent Liu process, the changes of $S_{t}$ and $X_{t}$ are also independent of each other.

The solution of Model (1) is

$$
\left\{\begin{array}{l}
Y_{t}=Y_{0} \exp (\gamma t) \\
S_{t}=S_{0} \exp \left(\mu_{1} t+\sigma_{1} C_{1 t}\right) \\
X_{t}=X_{0} \exp \left(\mu_{2} t+\sigma_{2} C_{2 t}\right)
\end{array}\right.
$$


whose inverse uncertainty distribution is

$\begin{cases}\Phi_{1 t}^{-1}(\alpha)=S_{0} \exp \left(\mu_{1} t+\frac{\sqrt{3} \sigma_{1} t}{\pi} \ln \frac{\alpha}{1-\alpha}\right), & S_{0} \geq 0, \sigma_{1}>0 \\ \Phi_{2 t}^{-1}(\alpha)=X_{0} \exp \left(\mu_{2} t+\frac{\sqrt{3} \sigma_{2} t}{\pi} \ln \frac{\alpha}{1-\alpha}\right), & X_{0} \geq 0, \sigma_{2}>0\end{cases}$

where $\Phi_{1 t}^{-1}(\alpha)$ and $\Phi_{2 t}^{-1}(\alpha)$ are, respectively, inverse uncertainty distribution of $S_{t}$ and $X_{t}$.

Firstly, from the holder's perspective, the payoff at time $T$ is

$\left[\frac{1}{T} \int_{0}^{T} S_{t} d t-\frac{1}{T} \int_{0}^{T} X_{t} d t\right]^{+}$

Let $f_{p c}^{a}$ denote the price of arithmetic Asian put 2 and call 1 option. Thus, at the initial moment, the holder of the option owns the net return

$-f_{p c}^{a}+\exp (-\gamma T)\left[\frac{1}{T} \int_{0}^{T} S_{t} d t-\frac{1}{T} \int_{0}^{T} X_{t} d t\right]^{+}$.

Secondly, from the seller's perspective, the payoff at time $T$ is

$-\left[\frac{1}{T} \int_{0}^{T} S_{t} d t-\frac{1}{T} \int_{0}^{T} X_{t} d t\right]^{+}$

Then, at the initial moment, the net return of the seller can be expressed:

$f_{p c}^{a}-\exp (-\gamma T)\left[\frac{1}{T} \int_{0}^{T} S_{t} d t-\frac{1}{T} \int_{0}^{T} X_{t} d t\right]^{+}$.

Both should own uniform expected payoff by the fair price principle, that is

$$
\begin{aligned}
& -f_{p c}^{a}+\exp (-\gamma T) E\left[\left(\frac{1}{T} \int_{0}^{T} S_{t} d t-\frac{1}{T} \int_{0}^{T} X_{t} d t\right)^{+}\right] \\
& =f_{p c}^{a}-\exp (-\gamma T) E\left[\left(\frac{1}{T} \int_{0}^{T} S_{t} d t-\frac{1}{T} \int_{0}^{T} X_{t} d t\right)^{+}\right] .
\end{aligned}
$$

From the above, we conclude that the price of arithmetic Asian put 2 and call 1 option is equal to the present value of the expected payoff.

Definition 3 Suppose that an arithmetic Asian put 2 and call 1 option for Model (1) has a maturity time $T$. Then, its price is defined by

$f_{p c}^{a}=\exp (-\gamma T) E\left[\left(\frac{1}{T} \int_{0}^{T} S_{t} d t-\frac{1}{T} \int_{0}^{T} X_{t} d t\right)^{+}\right]$
Theorem 7 Suppose that an arithmetic Asian put 2 and call 1 option for Model (1) has a maturity time T. Then, its price is

$$
\begin{aligned}
& f_{p c}^{a}=\exp (-\gamma T) \int_{0}^{1} \frac{1}{T}\left[\int_{0}^{T} S_{0} \exp \left(\mu_{1} t+\frac{\sqrt{3} \sigma_{1} t}{\pi} \ln \frac{\alpha}{1-\alpha}\right) d t\right. \\
& \left.-\int_{0}^{T} X_{0} \exp \left(\mu_{2} t+\frac{\sqrt{3} \sigma_{2} t}{\pi} \ln \frac{1-\alpha}{\alpha}\right) d t\right]^{+} d \alpha
\end{aligned}
$$

Proof We show that the uncertain variable

$\left[\frac{1}{T} \int_{0}^{T} S_{t} d t-\frac{1}{T} \int_{0}^{T} X_{t} d t\right]^{+}$

has an inverse uncertainty distribution

$\left[\frac{1}{T} \int_{0}^{T} S_{t}^{\alpha} d t-\frac{1}{T} \int_{0}^{T} X_{t}^{1-\alpha} d t\right]^{+}$

where

$\left\{\begin{array}{l}S_{t}^{\alpha}=S_{0} \exp \left(\mu_{1} t+\frac{\sqrt{3} \sigma_{1} t}{\pi} \ln \frac{\alpha}{1-\alpha}\right) \\ X_{t}^{1-\alpha}=X_{0} \exp \left(\mu_{2} t+\frac{\sqrt{3} \sigma_{2} t}{\pi} \ln \frac{1-\alpha}{\alpha}\right) .\end{array}\right.$

The uncertain processes $S_{t}$ and $X_{t}$ own inverse uncertainty distributions which are represented by Equation (2). By using Theorem 4 and Theorem 5, we derive that the inverse uncertainty distributions of the two time integrals

$\int_{0}^{T} S_{t} d t$ and $-\int_{0}^{\mathrm{T}} \mathrm{X}_{\mathrm{t}} \mathrm{dt}$

are

$\int_{0}^{T} S_{t}^{\alpha} d t$ and $\int_{0}^{\mathrm{T}} \mathrm{X}_{\mathrm{t}}^{1-} \mathrm{dt}$,

respectively.

It follows from Theorem 6 that the uncertain variable

$\left[\frac{1}{T} \int_{0}^{T} S_{t} d t-\frac{1}{T} \int_{0}^{T} X_{t} d t\right]^{+}$

has an inverse uncertainty distribution

$\left[\frac{1}{T} \int_{0}^{T} S_{t}^{\alpha} d t-\frac{1}{T} \int_{0}^{T} X_{t}^{1-\alpha} d t\right]^{+}$ 
Hence, the pricing formula is

$$
\begin{aligned}
f_{p c}^{a}= & \exp (-\gamma T) E\left[\left(\frac{1}{T} \int_{0}^{T} S_{t} d t-\frac{1}{T} \int_{0}^{T} X_{t} d t\right)^{+}\right] \\
= & \exp (-\gamma T) \int_{0}^{1}\left[\frac{1}{T} \int_{0}^{T} S_{t}^{\alpha} d t-\frac{1}{T} \int_{0}^{T} X_{t}^{1-\alpha} d t\right]^{+} d \alpha \\
= & \exp (-\gamma T) \int_{0}^{1} \frac{1}{T}\left[\int_{0}^{T} S_{0} \exp \left(\mu_{1} t+\frac{\sqrt{3} \sigma_{1} t}{\pi} \ln \frac{\alpha}{1-\alpha}\right) d t\right. \\
& \left.-\int_{0}^{T} X_{0} \exp \left(\mu_{2} t+\frac{\sqrt{3} \sigma_{2} t}{\pi} \ln \frac{1-\alpha}{\alpha}\right) d t\right]^{+} d \alpha \\
= & \exp (-\gamma T) \int_{0}^{1} \frac{1}{T}\left[\int_{0}^{T} S_{0} \exp \left(\mu_{1} t+\frac{\sqrt{3} \sigma_{1} t}{\pi} \ln \frac{\alpha}{1-\alpha}\right) d t\right. \\
& \left.-\int_{0}^{T} X_{0} \exp \left(\mu_{2} t+\frac{\sqrt{3} \sigma_{2} t}{\pi} \ln \frac{\alpha}{1-\alpha}\right) d t\right]^{+} d \alpha .
\end{aligned}
$$

The proof is completed.

Example 1 In Model (1), we assume that $\gamma=0.07, \mu_{1}=$ $0.02, \sigma_{1}=0.04, \mu_{2}=-0.01, \sigma_{2}=0.04$. Consider that there are $S_{0}=8, X_{0}=7, T=6$ for an arithmetic Asian put 2 and call 1 option. Then, $f_{p c}^{a}=1.1339$ by Theorem 7 .

Figure 1 demonstrates that the price $f_{p c}^{a}$ is an increasing function of maturity time $T$ if other parameters are invariant.

\subsection{Geometric Asian put 2 and call 1 option}

Geometric Asian put 2 and call 1 option is a kind of exchange option, in which market prices of Asset 1 and Asset 2 are calculated by geometric average method. The payoff of the option relies on the geometric mean of Asset 1 and Asset 2 market price within an agreed period of time. The geometric mean of $\mathrm{N}$ numbers is defined as $\sqrt[n]{S_{1} S_{2} \cdots S_{n}}$, namely

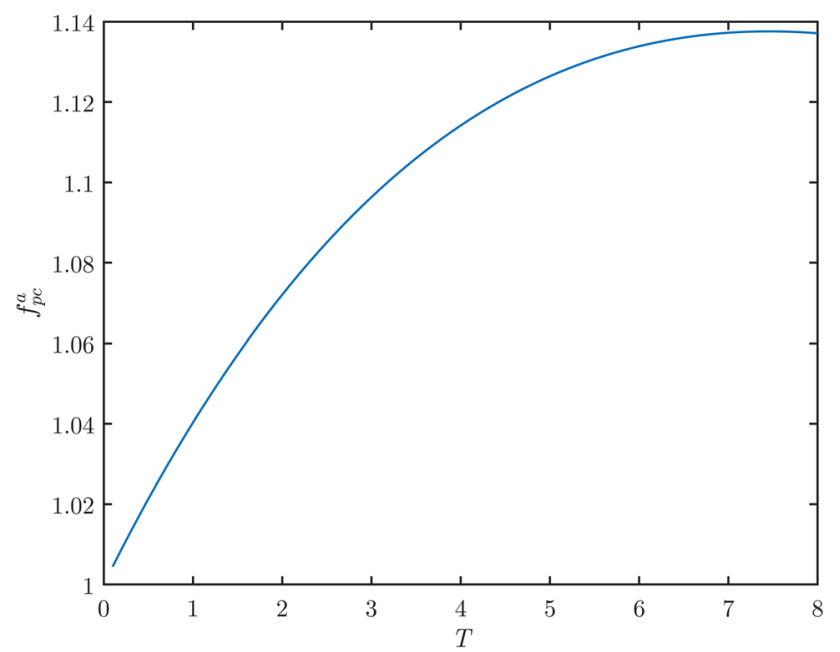

Fig. 1 price $f_{p c}^{a}$ concerning maturity time $T$ in Example 1 $\exp \left\{\left[\ln \left(S_{1}\right)+\ln \left(S_{2}\right)+\cdots+\ln \left(S_{n}\right)\right] / n\right\}$. When the underlying asset price $S_{t}$ changes continuously from time zero to time $T$, the geometric mean of $S_{t}$ can be written as $\exp \left(\int_{0}^{T} \ln \left(S_{t}\right) d t / T\right)$.

Assume that geometric Asian put 2 and call 1 option for Model (1) has a maturity time $T$.

Firstly, from the holder's perspective, the payoff at time $T$ is

$$
\begin{aligned}
& {\left[\exp \left(\frac{1}{T} \int_{0}^{T} \ln \left(S_{t}\right) d t\right)\right.} \\
& \left.\quad-\exp \left(\frac{1}{T} \int_{0}^{T} \ln \left(X_{t}\right) d t\right)\right]^{+} .
\end{aligned}
$$

Let $f_{p c}^{g}$ denote the price of geometric Asian put 2 and call 1 option. Thus, at the initial moment, the holder of the option owns the net return

$$
\begin{aligned}
& -f_{p c}^{g}+\exp (-\gamma T)\left[\exp \left(\frac{1}{T} \int_{0}^{T} \ln \left(S_{t}\right) d t\right)\right. \\
& \left.-\exp \left(\frac{1}{T} \int_{0}^{T} \ln \left(X_{t}\right) d t\right)\right]^{+} .
\end{aligned}
$$

Secondly, from the seller's perspective, the payoff at time $T$ is

$$
\begin{aligned}
& -\left[\exp \left(\frac{1}{T} \int_{0}^{T} \ln \left(S_{t}\right) d t\right)\right. \\
& \left.-\exp \left(\frac{1}{T} \int_{0}^{T} \ln \left(X_{t}\right) d t\right)\right]^{+} .
\end{aligned}
$$

Then, at the initial moment, the net return of the seller can be expressed:

$$
\begin{aligned}
& f_{p c}^{g}-\exp (-\gamma T)\left[\exp \left(\frac{1}{T} \int_{0}^{T} \ln \left(S_{t}\right) d t\right)\right. \\
& \left.-\exp \left(\frac{1}{T} \int_{0}^{T} \ln \left(X_{t}\right) d t\right)\right]^{+} .
\end{aligned}
$$

Both should own uniform expected payoff by the fair price principle, that is

$$
\begin{aligned}
& -f_{p c}^{g}+\exp (-\gamma T) E\left[\left(\exp \left(\frac{1}{T} \int_{0}^{T} \ln \left(S_{t}\right) d t\right)\right.\right. \\
& \left.\left.-\exp \left(\frac{1}{T} \int_{0}^{T} \ln \left(X_{t}\right) d t\right)\right)^{+}\right] \\
& =f_{p c}^{g}-\exp (-\gamma T) E\left[\left(\exp \left(\frac{1}{T} \int_{0}^{T} \ln \left(S_{t}\right) d t\right)\right.\right. \\
& \left.\left.-\exp \left(\frac{1}{T} \int_{0}^{T} \ln \left(X_{t}\right) d t\right)\right)^{+}\right] .
\end{aligned}
$$


From the above, we conclude that the price of geometric Asian put 2 and call 1 option is equal to the present value of the expected payoff.

Definition 4 Suppose that a geometric Asian put 2 and call 1 option for Model (1) has a maturity time $T$. Then, its price is defined by

$$
\begin{aligned}
& f_{p c}^{g}=\exp (-\gamma T) E\left[\left(\exp \left(\frac{1}{T} \int_{0}^{T} \ln \left(S_{t}\right) d t\right)\right.\right. \\
& \left.\left.-\exp \left(\frac{1}{T} \int_{0}^{T} \ln \left(X_{t}\right) d t\right)\right)^{+}\right] .
\end{aligned}
$$

Theorem 8 Suppose that a geometric Asian put 2 and call 1 option for Model (1) has a maturity time T. Then, its price is

$$
\begin{aligned}
& f_{p c}^{g}=\exp (-\gamma T) \int_{0}^{1}\left[S_{0} \exp \left(\frac{\mu_{1} T}{2}+\frac{\sqrt{3} \sigma_{1} T}{2 \pi} \ln \frac{\alpha}{1-\alpha}\right)\right. \\
& \left.-X_{0} \exp \left(\frac{\mu_{2} T}{2}+\frac{\sqrt{3} \sigma_{2} T}{2 \pi} \ln \frac{\alpha}{1-\alpha}\right)\right]^{+} d \alpha
\end{aligned}
$$

Proof We show that the uncertain variable

$$
\begin{aligned}
& {\left[\exp \left(\frac{1}{T} \int_{0}^{T} \ln \left(S_{t}\right) d t\right)\right.} \\
& \left.\quad-\exp \left(\frac{1}{T} \int_{0}^{T} \ln \left(X_{t}\right) d t\right)\right]^{+}
\end{aligned}
$$

has an inverse uncertainty distribution

$$
\begin{aligned}
& {\left[\exp \left(\frac{1}{T} \int_{0}^{T} \ln \left(S_{t}^{\alpha}\right) d t\right)\right.} \\
& \left.\quad-\exp \left(\frac{1}{T} \int_{0}^{T} \ln \left(X_{t}^{1-\alpha}\right) d t\right)\right]^{+}
\end{aligned}
$$

where

$$
\left\{\begin{array}{l}
S_{t}^{\alpha}=S_{0} \exp \left(\mu_{1} t+\frac{\sqrt{3} \sigma_{1} t}{\pi} \ln \frac{\alpha}{1-\alpha}\right) \\
X_{t}^{1-\alpha}=X_{0} \exp \left(\mu_{2} t+\frac{\sqrt{3} \sigma_{2} t}{\pi} \ln \frac{1-\alpha}{\alpha}\right) .
\end{array}\right.
$$

The uncertain processes $S_{t}$ and $X_{t}$ own inverse uncertainty distributions which are represented by Equation (2). Since $\exp \left(\int_{0}^{T} \ln \left(S_{t}\right) d t / T\right)$ is a continuous and strictly increasing function concerning $\ln (x)$, the inverse uncertainty distributions of the two time integrals

$$
\exp \left(\frac{1}{T} \int_{0}^{T} \ln \left(S_{t}\right) d t\right) \text { and }-\exp \left(\frac{1}{\mathrm{~T}} \int_{0}^{\mathrm{T}} \ln \left(\mathrm{X}_{\mathrm{t}}\right) \mathrm{dt}\right)
$$

are

$\exp \left(\frac{1}{T} \int_{0}^{T} \ln \left(S_{t}^{\alpha}\right) d t\right)$ and $-\exp \left(\frac{1}{\mathrm{~T}} \int_{0}^{\mathrm{T}} \ln \left(\mathrm{X}_{\mathrm{t}}^{1-}\right) \mathrm{dt}\right)$

following from Theorem 4 and Theorem 5, respectively.

By using Theorem 6, we derive that the uncertain variable

$$
\left[\exp \left(\frac{1}{T} \int_{0}^{T} \ln \left(S_{t}\right) d t\right)-\exp \left(\frac{1}{T} \int_{0}^{T} \ln \left(X_{t}\right) d t\right)\right]^{+}
$$

has an inverse uncertainty distribution

$$
\left[\exp \left(\frac{1}{T} \int_{0}^{T} \ln \left(S_{t}^{\alpha}\right) d t\right)-\exp \left(\frac{1}{T} \int_{0}^{T} \ln \left(X_{t}^{1-\alpha}\right) d t\right)\right]^{+}
$$

Hence, the pricing formula is

$$
\begin{aligned}
f_{p c}^{g}= & \exp (-\gamma T) E\left[\left(\exp \left(\frac{1}{T} \int_{0}^{T} \ln \left(S_{t}\right) d t\right)\right.\right. \\
& \left.\left.-\exp \left(\frac{1}{T} \int_{0}^{T} \ln \left(X_{t}\right) d t\right)\right)^{+}\right] \\
= & \exp (-\gamma T) \int_{0}^{1}\left[\exp \left(\frac{1}{T} \int_{0}^{T} \ln \left(S_{t}^{\alpha}\right) d t\right)\right. \\
& \left.-\exp \left(\frac{1}{T} \int_{0}^{T} \ln \left(X_{t}^{1-\alpha}\right) d t\right)\right]^{+} d \alpha \\
= & \exp (-\gamma T) \int_{0}^{1}\left[S_{0} \exp \left(\frac{\mu_{1} T}{2}+\frac{\sqrt{3} \sigma_{1} T}{2 \pi} \ln \frac{\alpha}{1-\alpha}\right)\right. \\
& \left.-X_{0} \exp \left(\frac{\mu_{2} T}{2}+\frac{\sqrt{3} \sigma_{2} T}{2 \pi} \ln \frac{1-\alpha}{\alpha}\right)\right]^{+} d \alpha \\
= & \exp (-\gamma T) \int_{0}^{1}\left[S_{0} \exp \left(\frac{\mu_{1} T}{2}+\frac{\sqrt{3} \sigma_{1} T}{2 \pi} \ln \frac{\alpha}{1-\alpha}\right)\right. \\
& \left.-X_{0} \exp \left(\frac{\mu_{2} T}{2}+\frac{\sqrt{3} \sigma_{2} T}{2 \pi} \ln \frac{\alpha}{1-\alpha}\right)\right]^{+} d \alpha .
\end{aligned}
$$

The proof is completed.

Example 2 In Model (1), we assume that $\gamma=0.02, \mu_{1}=$ $0.04, \sigma_{1}=0.06, \mu_{2}=-0.04, \sigma_{2}=0.05$. Consider that there are $S_{0}=8, X_{0}=7, T=6$ for a geometric Asian put 2 and call 1 option. Then $f_{p c}^{g}=1.0517$ by Theorem 8 .

Figure 2 demonstrates that the price $f_{p c}^{g}$ is an increasing function of maturity time $T$ if other parameters are invariant.

\section{Asian rainbow call option}

Asian rainbow call option is a contract that provides the holder with a right to purchase the maximum or minimum asset at a given price at maturity time. The gain of the option 


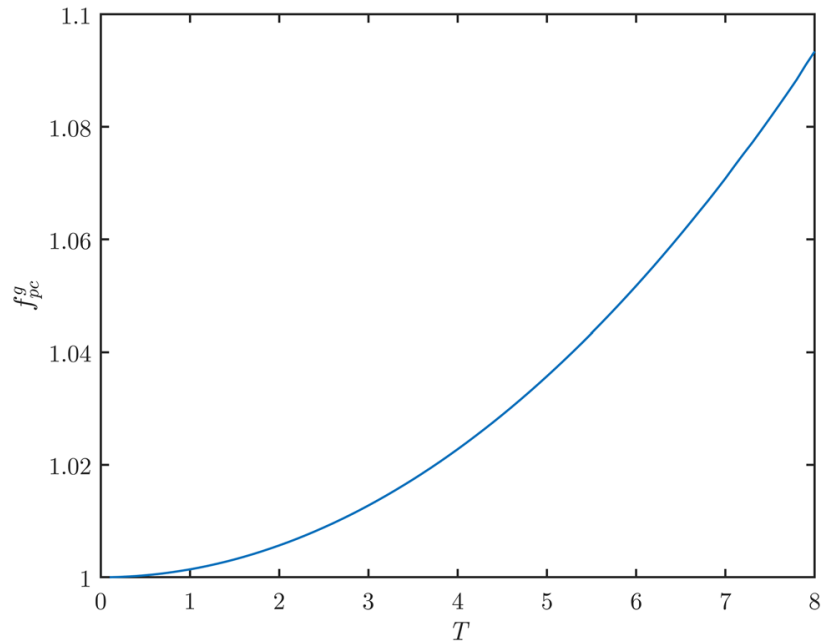

Fig. 2 price $f_{p c}^{g}$ concerning maturity time $T$ in Example 2

depends on the mean of the maximum or minimum underlying asset price over its lifetime. The means of underlying assets prices are calculated by the arithmetic average and geometric average methods. In this section, we study arithmetic Asian rainbow call on max option, geometric Asian rainbow call on max option, arithmetic Asian rainbow call on min option and geometric Asian rainbow call on min option. Furthermore, we obtain the pricing formulas of Asian rainbow call on max option and Asian rainbow call on min option by strict deduction.

\subsection{Arithmetic Asian rainbow call on max option}

Arithmetic Asian rainbow call on max option signifies that the holder can purchase maximum asset at the exercise price at the time of execution. The payoff of the option relies on the arithmetic mean of maximum asset price within an agreed period of time.

Considering that the underlying asset prices of arithmetic Asian rainbow call on max option with a maturity time $T$ and an exercise price $K$ obey different uncertain differential equations, the following model is proposed:

$$
\left\{\begin{array}{l}
d Y_{t}=\gamma Y_{t} d t \\
d S_{1 t}=\mu_{1} S_{1 t} d t+\sigma_{1} S_{1 t} d C_{1 t} \\
d S_{2 t}=\mu_{2} S_{2 t} d t+\sigma_{2} S_{2 t} d C_{2 t} \\
\vdots \\
d S_{n t}=\mu_{n} S_{n t} d t+\sigma_{n} S_{n t} d C_{n t}
\end{array}\right.
$$

where $Y_{t}$ is the bond price with riskless interest rate $\gamma, S_{i t}$ is the price of Asset $i$ at time $t, C_{i t}$ are independent Liu processes, $\sigma_{i}$ are the log-diffusion of $S_{i t}$, and $\mu_{i}$ are the logdrift of $S_{i t},(i=1,2, \ldots, n)$.
Since $C_{i t}$ are independent Liu process, the changes of $S_{i t}$ are also independent of each other.

The solution of Model (3) is

$$
\left\{\begin{array}{l}
Y_{t}=Y_{0} \exp (\gamma t) \\
S_{1 t}=S_{10} \exp \left(\mu_{1} t+\sigma_{1} C_{1 t}\right) \\
S_{2 t}=S_{20} \exp \left(\mu_{2} t+\sigma_{2} C_{2 t}\right) \\
\vdots \\
S_{n t}=S_{n 0} \exp \left(\mu_{n} t+\sigma_{n} C_{n t}\right)
\end{array}\right.
$$

whose inverse uncertainty distribution is

$$
\left\{\begin{array}{cl}
\Phi_{1 t}^{-1}(\alpha)=S_{10} \exp \left(\mu_{1} t+\frac{\sqrt{3} \sigma_{1} t}{\pi} \ln \frac{\alpha}{1-\alpha}\right), & S_{10} \geq 0, \sigma_{1}>0 \\
\Phi_{2 t}^{-1}(\alpha)=S_{20} \exp \left(\mu_{2} t+\frac{\sqrt{3} \sigma_{2} t}{\pi} \ln \frac{\alpha}{1-\alpha}\right), & S_{20} \geq 0, \sigma_{2}>0 \\
\vdots & \\
\Phi_{n t}^{-1}(\alpha)=S_{n 0} \exp \left(\mu_{n} t+\frac{\sqrt{3} \sigma_{n} t}{\pi} \ln \frac{\alpha}{1-\alpha}\right), & S_{n 0} \geq 0, \sigma_{n}>0
\end{array}\right.
$$

where $\Phi_{i t}^{-1}(\alpha)$ are inverse uncertainty distribution of $S_{i t}$.

Firstly, from the holder's perspective, the payoff at time $T$ is

$\left[\max _{1 \leq i \leq n}\left(\frac{1}{T} \int_{0}^{T} S_{i t} d t\right)-K\right]^{+}$

Let $f_{1 c}^{a}$ be the price of arithmetic Asian rainbow call on max option. Thus, at the initial moment, the holder of the option owns the net return

$-f_{1 c}^{a}+\exp (-\gamma T)\left[\max _{1 \leq i \leq n}\left(\frac{1}{T} \int_{0}^{T} S_{i t} d t\right)-K\right]^{+}$.

Secondly, from the seller's perspective, the payoff at time $T$ is

$-\left[\max _{1 \leq i \leq n}\left(\frac{1}{T} \int_{0}^{T} S_{i t} d t\right)-K\right]^{+}$.

Then, at the initial moment, the net return of the seller can be expressed:

$$
f_{1 c}^{a}-\exp (-\gamma T)\left[\max _{1 \leq i \leq n}\left(\frac{1}{T} \int_{0}^{T} S_{i t} d t\right)-K\right]^{+}
$$

Both should own uniform expected payoff by the fair price principle, that is

$$
\begin{aligned}
& -f_{1 c}^{a}+\exp (-\gamma T) E\left[\left(\max _{1 \leq i \leq n}\left(\frac{1}{T} \int_{0}^{T} S_{i t} d t\right)-K\right)^{+}\right] \\
& =f_{1 c}^{a}-\exp (-\gamma T) E\left[\left(\max _{1 \leq i \leq n}\left(\frac{1}{T} \int_{0}^{T} S_{i t} d t\right)-K\right)^{+}\right] .
\end{aligned}
$$


From the above, we conclude that the price of arithmetic Asian rainbow call on max option is equal to the present value of the expected payoff.

Definition 5 Suppose that an arithmetic Asian rainbow call on max option for Model (3) has a maturity time $T$ and an exercise price $K$. Then, its price is defined by

$$
f_{1 c}^{a}=\exp (-\gamma T) E\left[\left(\max _{1 \leq i \leq n}\left(\frac{1}{T} \int_{0}^{T} S_{i t} d t\right)-K\right)^{+}\right]
$$

Theorem 9 Suppose that an arithmetic Asian rainbow call on max option for Model (3) has a maturity time $T$ and an exercise price $K$. Then, its price is

$$
\begin{aligned}
& f_{1 c}^{a}=\exp (-\gamma T) \int_{0}^{1}\left[\operatorname { m a x } _ { 1 \leq i \leq n } \left(\frac{S_{i 0}}{T} \int_{0}^{T}\right.\right. \\
& \left.\left.\exp \left(\mu_{i} t+\frac{\sqrt{3} \sigma_{i} t}{\pi} \ln \frac{\alpha}{1-\alpha}\right) d t\right)-K\right]^{+} d \alpha .
\end{aligned}
$$

Proof We show that the uncertain variable

$$
\left[\max _{1 \leq i \leq n}\left(\frac{1}{T} \int_{0}^{T} S_{i t} d t\right)-K\right]^{+}
$$

has an inverse uncertainty distribution

$\left[\max _{1 \leq i \leq n}\left(\frac{1}{T} \int_{0}^{T} S_{i t}^{\alpha} d t\right)-K\right]^{+}$

where

$S_{i t}^{\alpha}=S_{i 0} \exp \left(\mu_{i} t+\frac{\sqrt{3} \sigma_{i} t}{\pi} \ln \frac{\alpha}{1-\alpha}\right)$.

Since $\int_{0}^{T} x d t / T$ is an increasing function with regard to $x$, we derive

$$
\begin{aligned}
& \left\{\left[\max _{1 \leq i \leq n}\left(\frac{1}{T} \int_{0}^{T} S_{i t} d t\right)-K\right]^{+}\right. \\
& \left.\quad \leq\left[\max _{1 \leq i \leq n}\left(\frac{1}{T} \int_{0}^{T} S_{i t}^{\alpha} d t\right)-K\right]^{+}\right\} \\
& \quad \supseteq\left\{\max _{1 \leq i \leq n}\left(\frac{1}{T} \int_{0}^{T} S_{i t} d t\right)\right. \\
& \left.\quad \leq \max _{1 \leq i \leq n}\left(\frac{1}{T} \int_{0}^{T} S_{i t}^{\alpha} d t\right), t \in[0, T]\right\} \\
& \quad \supseteq\left\{S_{i t} \leq S_{i t}^{\alpha}, t \in[0, T], 1 \leq i \leq n\right\}
\end{aligned}
$$

and

$$
\begin{aligned}
& \left\{\left[\max _{1 \leq i \leq n}\left(\frac{1}{T} \int_{0}^{T} S_{i t} d t\right)-K\right]^{+}\right. \\
& \left.>\left[\max _{1 \leq i \leq n}\left(\frac{1}{T} \int_{0}^{T} S_{i t}^{\alpha} d t\right)-K\right]^{+}\right\} \\
& \quad \supseteq\left\{\max _{1 \leq i \leq n}\left(\frac{1}{T} \int_{0}^{T} S_{i t} d t\right)\right. \\
& \left.\quad>\max _{1 \leq i \leq n}\left(\frac{1}{T} \int_{0}^{T} S_{i t}^{\alpha} d t\right), t \in[0, T]\right\} \\
& \supseteq\left\{S_{i t}>S_{i t}^{\alpha}, t \in[0, T], 1 \leq i \leq n\right\} .
\end{aligned}
$$

From Theorem 1 and Theorem 4, we have

$$
\begin{aligned}
\mathcal{M} & \left\{\left[\max _{1 \leq i \leq n}\left(\frac{1}{T} \int_{0}^{T} S_{i t} d t\right)-K\right]^{+}\right. \\
& \left.\leq\left[\max _{1 \leq i \leq n}\left(\frac{1}{T} \int_{0}^{T} S_{i t}^{\alpha} d t\right)-K\right]^{+}\right\} \\
\geq & \mathcal{M}\left\{S_{i t} \leq S_{i t}^{\alpha}, t \in[0, T], 1 \leq i \leq n\right\} \\
& =\mathcal{M}\left\{\bigcap_{i=1}^{n}\left(S_{i t} \leq S_{i t}^{\alpha}\right), t \in[0, T]\right\} \\
& =\bigwedge_{i=1} \mathcal{M}\left\{S_{i t} \leq S_{i t}^{\alpha}, t \in[0, T]\right\} \\
& =\alpha
\end{aligned}
$$

and

$$
\begin{aligned}
\mathcal{M} & \left\{\left[\max _{1 \leq i \leq n}\left(\frac{1}{T} \int_{0}^{T} S_{i t} d t\right)-K\right]^{+}\right. \\
> & {\left.\left[\max _{1 \leq i \leq n}\left(\frac{1}{T} \int_{0}^{T} S_{i t}^{\alpha} d t\right)-K\right]^{+}\right\} } \\
\geq & \mathcal{M}\left\{S_{i t}>S_{i t}^{\alpha}, t \in[0, T], 1 \leq i \leq n\right\} \\
& =\mathcal{M}\left\{\bigcap_{i=1}^{n}\left(S_{i t}>S_{i t}^{\alpha}\right), t \in[0, T]\right\} \\
& =\bigwedge \mathfrak{M}\left\{S_{i t}>S_{i t}^{\alpha}, t \in[0, T]\right\} \\
& \bigwedge_{i=1} \\
= & 1-\alpha .
\end{aligned}
$$


We can obtain

$$
\begin{aligned}
\mathcal{M} & \left\{\left[\max _{1 \leq i \leq n}\left(\frac{1}{T} \int_{0}^{T} S_{i t} d t\right)-K\right]^{+}\right. \\
\leq & {\left.\left[\max _{1 \leq i \leq n}\left(\frac{1}{T} \int_{0}^{T} S_{i t}^{\alpha} d t\right)-K\right]^{+}\right\} } \\
& +\mathcal{M}\left\{\left[\max _{1 \leq i \leq n}\left(\frac{1}{T} \int_{0}^{T} S_{i t} d t\right)-K\right]^{+}\right. \\
> & {\left.\left[\max _{1 \leq i \leq n}\left(\frac{1}{T} \int_{0}^{T} S_{i t}^{\alpha} d t\right)-K\right]^{+}\right\} } \\
= & 1
\end{aligned}
$$

by using duality axiom.

It follows from Eqs. (4)-(6) that

$$
\begin{aligned}
\mathcal{M} & \left\{\left[\max _{1 \leq i \leq n}\left(\frac{1}{T} \int_{0}^{T} S_{i t} d t\right)-K\right]^{+}\right. \\
& \left.\leq\left[\max _{1 \leq i \leq n}\left(\frac{1}{T} \int_{0}^{T} S_{i t}^{\alpha} d t\right)-K\right]^{+}\right\} \\
& =\alpha .
\end{aligned}
$$

Thus, the uncertain variable

$$
\left[\max _{1 \leq i \leq n}\left(\frac{1}{T} \int_{0}^{T} S_{i t} d t\right)-K\right]^{+}
$$

has an inverse uncertainty distribution

$$
\left[\max _{1 \leq i \leq n}\left(\frac{1}{T} \int_{0}^{T} S_{i t}^{\alpha} d t\right)-K\right]^{+}
$$

Hence, the pricing formula is

$$
\begin{aligned}
f_{1 c}^{a}= & \exp (-\gamma T) E\left[\left(\max _{1 \leq i \leq n}\left(\frac{1}{T} \int_{0}^{T} S_{i t} d t\right)-K\right)^{+}\right] \\
= & \exp (-\gamma T) \int_{0}^{1}\left[\max _{1 \leq i \leq n}\left(\frac{1}{T} \int_{0}^{T} S_{i t}^{\alpha} d t\right)-K\right]^{+} d \alpha \\
= & \exp (-\gamma T) \int_{0}^{1}\left[\operatorname { m a x } _ { 1 \leq i \leq n } \left(\frac{S_{i 0}}{T} \int_{0}^{T}\right.\right. \\
& \left.\left.\exp \left(\mu_{i} t+\frac{\sqrt{3} \sigma_{i} t}{\pi} \ln \frac{\alpha}{1-\alpha}\right) d t\right)-K\right]^{+} d \alpha .
\end{aligned}
$$

The proof is completed.

Example 3 Consider that there are $S_{10}=7, S_{20}=6, K=6$, $T=5$ for an arithmetic Asian rainbow call on max option with two underlying stocks. In Model (3), we assume that $\gamma=0.07, \mu_{1}=0.02, \sigma_{1}=0.06, \mu_{2}=0.04, \sigma_{2}=0.06$.

Then, $f_{1 c}^{a}=1.0411$ by Theorem 9 .

Figure 3 demonstrates that the price $f_{1 c}^{a}$ is an increasing function of maturity time $T$ if other parameters are invariant.

\subsection{Geometric Asian rainbow call on max option}

Geometric Asian rainbow call on max option signifies that the holder can purchase maximum asset at the exercise price at the time of execution. The payoff of the option relies on the geometric mean of maximum asset price within an agreed period of time.

Assume that geometric Asian rainbow call on max option possesses a maturity time $T$ and an exercise price $K$ for Model (3).

Firstly, from the holder's perspective, the payoff at time $T$ is

$\left[\max _{1 \leq i \leq n}\left(\exp \left(\frac{1}{T} \int_{0}^{T} \ln \left(S_{i t}\right) d t\right)\right)-K\right]^{+}$

Let $f_{1 c}^{g}$ be the price of geometric Asian rainbow call on max option. Thus, at the initial moment, the holder of the option owns the net return

$-f_{1 c}^{g}+\exp (-\gamma T)\left[\max _{1 \leq i \leq n}\left(\exp \left(\frac{1}{T} \int_{0}^{T} \ln \left(S_{i t}\right) d t\right)\right)-K\right]^{+}$.

Secondly, from the seller's perspective, the payoff at time $T$ is

$-\left[\max _{1 \leq i \leq n}\left(\exp \left(\frac{1}{T} \int_{0}^{T} \ln \left(S_{i t}\right) d t\right)\right)-K\right]^{+}$

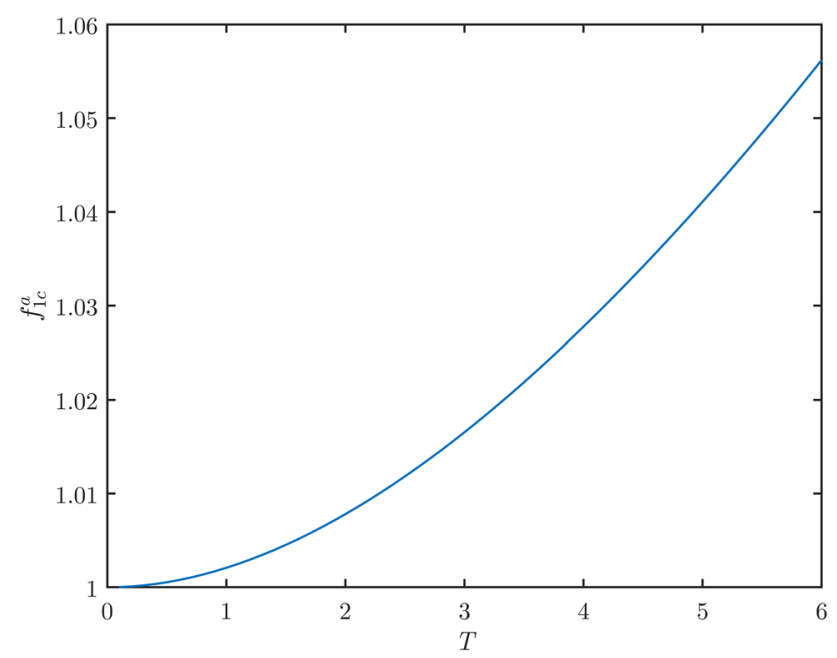

Fig. 3 price $f_{1 c}^{a}$ concerning maturity time $T$ in Example 3 
Then, at the initial moment, the net return of the seller can be expressed:

$$
f_{1 c}^{g}-\exp (-\gamma T)\left[\max _{1 \leq i \leq n}\left(\exp \left(\frac{1}{T} \int_{0}^{T} \ln \left(S_{i t}\right) d t\right)\right)-K\right]^{+}
$$

Both should own uniform expected payoff by the fair price principle, that is

$$
\begin{aligned}
& -f_{1 c}^{g}+\exp (-\gamma T) E\left[\left(\max _{1 \leq i \leq n}\left(\exp \left(\frac{1}{T} \int_{0}^{T} \ln \left(S_{i t}\right) d t\right)\right)\right.\right. \\
& \left.-K)^{+}\right] \\
& =f_{1 c}^{g}-\exp (-\gamma T) E\left[\left(\max _{1 \leq i \leq n}\left(\exp \left(\frac{1}{T} \int_{0}^{T} \ln \left(S_{i t}\right) d t\right)\right)\right.\right. \\
& \left.-K)^{+}\right] .
\end{aligned}
$$

From the above, we conclude that the price of geometric Asian rainbow call on max option is equal to the present value of the expected payoff.

Definition 6 Suppose that a geometric Asian rainbow call on max option for Model (3) has a maturity time $\mathrm{T}$ and an exercise price $K$. Then, its price is defined by

$$
\begin{aligned}
& f_{1 c}^{g}=\exp (-\gamma T) E\left[\left(\max _{1 \leq i \leq n}\left(\exp \left(\frac{1}{T} \int_{0}^{T} \ln \left(S_{i t}\right) d t\right)\right)\right.\right. \\
& \left.-K)^{+}\right] .
\end{aligned}
$$

Theorem 10 Suppose that a geometric Asian rainbow call on max option for Model (3) has a maturity time $T$ and an exercise price $K$. Then, its price is

$$
\begin{aligned}
& f_{1 c}^{g}=\exp (-\gamma T) \int_{0}^{1}\left[\operatorname { m a x } _ { 1 \leq i \leq n } \left(S_{i 0}\right.\right. \\
& \left.\left.\quad \exp \left(\frac{\mu_{i} T}{2}+\frac{\sqrt{3} \sigma_{i} T}{2 \pi} \ln \frac{\alpha}{1-\alpha}\right)\right)-K\right]^{+} d \alpha .
\end{aligned}
$$

Proof We show that the uncertain variable

$$
\left[\max _{1 \leq i \leq n}\left(\exp \left(\frac{1}{T} \int_{0}^{T} \ln \left(S_{i t}\right) d t\right)\right)-K\right]^{+}
$$

has an inverse uncertainty distribution

$$
\left[\max _{1 \leq i \leq n}\left(\exp \left(\frac{1}{T} \int_{0}^{T} \ln \left(S_{i t}^{\alpha}\right) d t\right)\right)-K\right]^{+}
$$

where

$$
S_{i t}^{\alpha}=S_{i 0} \exp \left(\mu_{i} t+\frac{\sqrt{3} \sigma_{i} t}{\pi} \ln \frac{\alpha}{1-\alpha}\right) .
$$

Since exp $\left(\int_{0}^{T} \ln \left(S_{t}\right) d t / T\right)$ is an increasing function with regard to $\ln (x)$, we derive

$$
\begin{aligned}
& \left\{\left[\max _{1 \leq i \leq n}\left(\exp \left(\frac{1}{T} \int_{0}^{T} \ln \left(S_{i t}\right) d t\right)\right)-K\right]^{+}\right. \\
& \left.\quad \leq\left[\max _{1 \leq i \leq n}\left(\exp \left(\frac{1}{T} \int_{0}^{T} \ln \left(S_{i t}^{\alpha}\right) d t\right)\right)-K\right]^{+}\right\} \\
& \quad \supseteq\left\{\max _{1 \leq i \leq n}\left(\exp \left(\frac{1}{T} \int_{0}^{T} \ln \left(S_{i t}\right) d t\right)\right)\right. \\
& \left.\quad \leq \max _{1 \leq i \leq n}\left(\exp \left(\frac{1}{T} \int_{0}^{T} \ln \left(S_{i t}^{\alpha}\right) d t\right)\right), t \in[0, T]\right\} \\
& \quad \supseteq\left\{S_{i t} \leq S_{i t}^{\alpha}, t \in[0, T], 1 \leq i \leq n\right\}
\end{aligned}
$$

and

$$
\begin{aligned}
& \left\{\left[\max _{1 \leq i \leq n}\left(\exp \left(\frac{1}{T} \int_{0}^{T} \ln \left(S_{i t}\right) d t\right)\right)-K\right]^{+}\right. \\
& \left.>\left[\max _{1 \leq i \leq n}\left(\exp \left(\frac{1}{T} \int_{0}^{T} \ln \left(S_{i t}^{\alpha}\right) d t\right)\right)-K\right]^{+}\right\} \\
& \supseteq\left\{\max _{1 \leq i \leq n}\left(\exp \left(\frac{1}{T} \int_{0}^{T} \ln \left(S_{i t}\right) d t\right)\right)\right. \\
& \left.>\max _{1 \leq i \leq n}\left(\exp \left(\frac{1}{T} \int_{0}^{T} \ln \left(S_{i t}^{\alpha}\right) d t\right)\right), t \in[0, T]\right\} \\
& \supseteq\left\{S_{i t}>S_{i t}^{\alpha}, t \in[0, T], 1 \leq i \leq n\right\} .
\end{aligned}
$$

From Theorem 1 and Theorem 4, we have

$$
\begin{aligned}
\mathcal{M} & \left\{\left[\max _{1 \leq i \leq n}\left(\exp \left(\frac{1}{T} \int_{0}^{T} \ln \left(S_{i t}\right) d t\right)\right)-K\right]^{+}\right. \\
& \left.\leq\left[\max _{1 \leq i \leq n}\left(\exp \left(\frac{1}{T} \int_{0}^{T} \ln \left(S_{i t}^{\alpha}\right) d t\right)\right)-K\right]^{+}\right\} \\
\geq & \mathcal{M}\left\{S_{i t} \leq S_{i t}^{\alpha}, t \in[0, T], 1 \leq i \leq n\right\} \\
& =\mathcal{M}\left\{\bigcap_{i=1}^{n}\left(S_{i t} \leq S_{i t}^{\alpha}\right), t \in[0, T]\right\} \\
& =\bigwedge \\
& \bigwedge \\
& \mathcal{M}\left\{S_{i t} \leq S_{i t}^{\alpha}, t \in[0, T]\right\} \\
& =\alpha
\end{aligned}
$$


and

$$
\begin{aligned}
& \mathcal{M}\left\{\left[\max _{1 \leq i \leq n}\left(\exp \left(\frac{1}{T} \int_{0}^{T} \ln \left(S_{i t}\right) d t\right)\right)-K\right]^{+}\right. \\
& \left.>\quad\left[\max _{1 \leq i \leq n}\left(\exp \left(\frac{1}{T} \int_{0}^{T} \ln \left(S_{i t}^{\alpha}\right) d t\right)\right)-K\right]^{+}\right\} \\
& \geq \mathcal{M}\left\{S_{i t}>S_{i t}^{\alpha}, t \in[0, T], 1 \leq i \leq n\right\} \\
& =\mathcal{M}\left\{\bigcap_{i=1}^{n}\left(S_{i t}>S_{i t}^{\alpha}\right), t \in[0, T]\right\} \\
& \quad=\bigwedge_{i=1}^{n} \mathcal{N}\left\{S_{i t}>S_{i t}^{\alpha}, t \in[0, T]\right\} \\
& \quad=1-\alpha .
\end{aligned}
$$

We can obtain

$$
\begin{aligned}
\mathcal{M} & \left\{\left[\max _{1 \leq i \leq n}\left(\exp \left(\frac{1}{T} \int_{0}^{T} \ln \left(S_{i t}\right) d t\right)\right)-K\right]^{+}\right. \\
\leq & {\left.\left[\max _{1 \leq i \leq n}\left(\exp \left(\frac{1}{T} \int_{0}^{T} \ln \left(S_{i t}^{\alpha}\right) d t\right)\right)-K\right]^{+}\right\} } \\
& +\mathcal{M}\left\{\left[\max _{1 \leq i \leq n}\left(\exp \left(\frac{1}{T} \int_{0}^{T} \ln \left(S_{i t}\right) d t\right)\right)-K\right]^{+}\right. \\
> & {\left.\left[\max _{1 \leq i \leq n}\left(\exp \left(\frac{1}{T} \int_{0}^{T} \ln \left(S_{i t}^{\alpha}\right) d t\right)\right)-K\right]^{+}\right\} } \\
= & 1
\end{aligned}
$$

by using duality axiom.

It follows from Eqs. (7)-(9) that

$$
\begin{aligned}
& \mathcal{M}\left\{\left[\max _{1 \leq i \leq n}\left(\exp \left(\frac{1}{T} \int_{0}^{T} \ln \left(S_{i t}\right) d t\right)\right)-K\right]^{+}\right. \\
& \left.\leq\left[\max _{1 \leq i \leq n}\left(\exp \left(\frac{1}{T} \int_{0}^{T} \ln \left(S_{i t}^{\alpha}\right) d t\right)\right)-K\right]^{+}\right\}=\alpha .
\end{aligned}
$$

Thus, the uncertain variable

$$
\left[\max _{1 \leq i \leq n}\left(\exp \left(\frac{1}{T} \int_{0}^{T} \ln \left(S_{i t}\right) d t\right)\right)-K\right]^{+}
$$

has an inverse uncertainty distribution

$$
\left[\max _{1 \leq i \leq n}\left(\exp \left(\frac{1}{T} \int_{0}^{T} \ln \left(S_{i t}^{\alpha}\right) d t\right)\right)-K\right]^{+}
$$

Hence, the pricing formula is

$$
\begin{aligned}
f_{1 c}^{g}= & \exp (-\gamma T) E\left[\left(\max _{1 \leq i \leq n}\left(\exp \left(\frac{1}{T} \int_{0}^{T} \ln \left(S_{i t}\right) d t\right)\right)-K\right)^{+}\right] \\
= & \exp (-\gamma T) \int_{0}^{1}\left[\max _{1 \leq i \leq n}\left(\exp \left(\frac{1}{T} \int_{0}^{T} \ln \left(S_{i t}^{\alpha}\right) d t\right)\right)-K\right]^{+} d \alpha \\
= & \exp (-\gamma T) \int_{0}^{1}\left[\max _{1 \leq i \leq n}\left(S_{i 0} \exp \left(\frac{\mu_{i} T}{2}+\frac{\sqrt{3} \sigma_{i} T}{2 \pi} \ln \frac{\alpha}{1-\alpha}\right)\right)\right. \\
& -K]^{+} d \alpha .
\end{aligned}
$$

The proof is completed.

Example 4 Consider that there are $S_{10}=7, S_{20}=6, K=6$, $T=5$ for a geometric Asian rainbow call on max option with two underlying stocks. In Model (3), we assume that $\gamma=0.07, \mu_{1}=0.02, \sigma_{1}=0.06, \mu_{2}=0.04, \sigma_{2}=0.06$. Then, $f_{1 c}^{g}=1.0164$ by Theorem 10 .

Figure 4 demonstrates that the price $f_{1 c}^{g}$ is an increasing function of maturity time $T$ if other parameters are invariant.

\subsection{Arithmetic Asian rainbow call on min option}

Arithmetic Asian rainbow call on min option signifies that the holder can purchase minimum asset at the exercise price at the time of execution. The payoff of the option relies on the arithmetic mean of minimum asset price within an agreed period of time.

Assume that arithmetic Asian rainbow call on min option possesses a maturity time $T$ and an exercise price $K$ for Model (3).

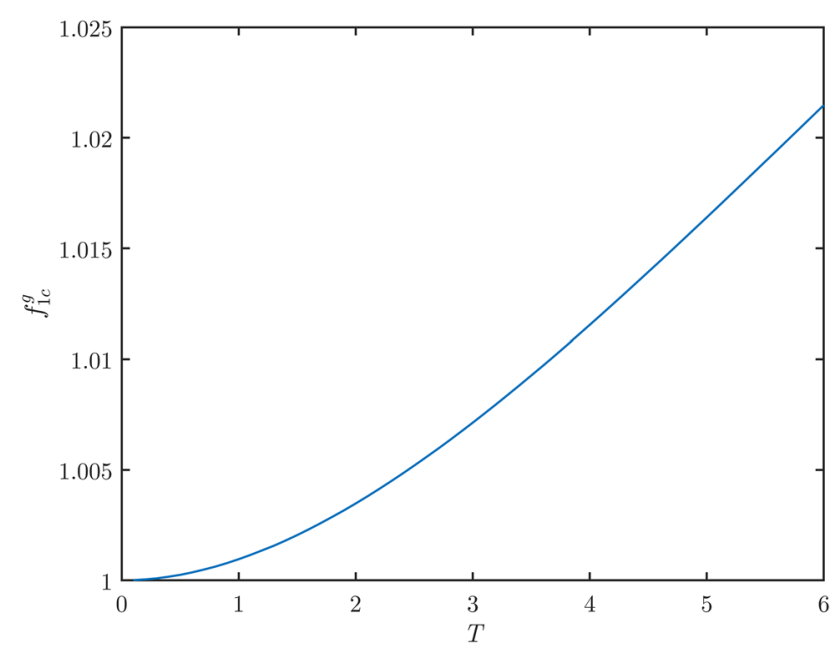

Fig. 4 price $f_{1 c}^{g}$ concerning maturity time $T$ in Example 4 
Firstly, from the holder's perspective, the payoff at time $T$ is

$\left[\min _{1 \leq i \leq n}\left(\frac{1}{T} \int_{0}^{T} S_{i t} d t\right)-K\right]^{+}$

Let $f_{2 c}^{a}$ be the price of arithmetic Asian rainbow call on min option. Thus, at the initial moment, the holder of the option owns the net return

$-f_{2 c}^{a}+\exp (-\gamma T)\left[\min _{1 \leq i \leq n}\left(\frac{1}{T} \int_{0}^{T} S_{i t} d t\right)-K\right]^{+}$

Secondly, from the seller's perspective, the payoff at time $T$ is

$-\left[\min _{1 \leq i \leq n}\left(\frac{1}{T} \int_{0}^{T} S_{i t} d t\right)-K\right]^{+}$.

Then, at the initial moment, the net return of the seller can be expressed:

$f_{2 c}^{a}-\exp (-\gamma T)\left[\min _{1 \leq i \leq n}\left(\frac{1}{T} \int_{0}^{T} S_{i t} d t\right)-K\right]^{+}$.

Both should own uniform expected payoff by the fair price principle, that is

$$
\begin{aligned}
& -f_{2 c}^{a}+\exp (-\gamma T) E\left[\left(\min _{1 \leq i \leq n}\left(\frac{1}{T} \int_{0}^{T} S_{i t} d t\right)-K\right)^{+}\right] \\
& =f_{2 c}^{a}-\exp (-\gamma T) E\left[\left(\min _{1 \leq i \leq n}\left(\frac{1}{T} \int_{0}^{T} S_{i t} d t\right)-K\right)^{+}\right] .
\end{aligned}
$$

From the above, we conclude that the price of arithmetic Asian rainbow call on min option is equal to the present value of the expected payoff.

Definition 7 Suppose that an arithmetic Asian rainbow call on min option for Model (3) has a maturity time $T$ and an exercise price $K$. Then, its price is defined by

$f_{2 c}^{a}=\exp (-\gamma T) E\left[\left(\min _{1 \leq i \leq n}\left(\frac{1}{T} \int_{0}^{T} S_{i t} d t\right)-K\right)^{+}\right]$.

Theorem 11 Suppose that an arithmetic Asian rainbow call on min option for Model (3) has a maturity time $T$ and an exercise price $K$. Then, its price is

$$
\begin{aligned}
& f_{2 c}^{a}=\exp (-\gamma T) \int_{0}^{1}\left[\operatorname { m i n } _ { 1 \leq i \leq n } \left(\frac{S_{i 0}}{T} \int_{0}^{T}\right.\right. \\
& \left.\left.\exp \left(\mu_{i} t+\frac{\sqrt{3} \sigma_{i} t}{\pi} \ln \frac{\alpha}{1-\alpha}\right) d t\right)-K\right]^{+} d \alpha .
\end{aligned}
$$

Proof We show that the uncertain variable

$\left[\min _{1 \leq i \leq n}\left(\frac{1}{T} \int_{0}^{T} S_{i t} d t\right)-K\right]^{+}$

has an inverse uncertainty distribution

$\left[\min _{1 \leq i \leq n}\left(\frac{1}{T} \int_{0}^{T} S_{i t}^{\alpha} d t\right)-K\right]^{+}$

where

$S_{i t}^{\alpha}=S_{i 0} \exp \left(\mu_{i} t+\frac{\sqrt{3} \sigma_{i} t}{\pi} \ln \frac{\alpha}{1-\alpha}\right)$.

Since $\int_{0}^{T} x d t / T$ is an increasing function with regard to $x$, we derive

$$
\begin{aligned}
& \left\{\left[\min _{1 \leq i \leq n}\left(\frac{1}{T} \int_{0}^{T} S_{i t} d t\right)-K\right]^{+}\right. \\
& \left.\quad \leq\left[\min _{1 \leq i \leq n}\left(\frac{1}{T} \int_{0}^{T} S_{i t}^{\alpha} d t\right)-K\right]^{+}\right\} \\
& \quad \supseteq\left\{\min _{1 \leq i \leq n}\left(\frac{1}{T} \int_{0}^{T} S_{i t} d t\right)\right. \\
& \left.\quad \leq \min _{1 \leq i \leq n}\left(\frac{1}{T} \int_{0}^{T} S_{i t}^{\alpha} d t\right), t \in[0, T]\right\} \\
& \supseteq\left\{S_{i t} \leq S_{i t}^{\alpha}, t \in[0, T], 1 \leq i \leq n\right\}
\end{aligned}
$$

and

$$
\begin{aligned}
& \left\{\left[\min _{1 \leq i \leq n}\left(\frac{1}{T} \int_{0}^{T} S_{i t} d t\right)-K\right]^{+}\right. \\
& \left.\quad>\left[\min _{1 \leq i \leq n}\left(\frac{1}{T} \int_{0}^{T} S_{i t}^{\alpha} d t\right)-K\right]^{+}\right\} \\
& \quad \supseteq\left\{\min _{1 \leq i \leq n}\left(\frac{1}{T} \int_{0}^{T} S_{i t} d t\right)\right. \\
& \left.\quad>\min _{1 \leq i \leq n}\left(\frac{1}{T} \int_{0}^{T} S_{i t}^{\alpha} d t\right), t \in[0, T]\right\} \\
& \quad \supseteq\left\{S_{i t}>S_{i t}^{\alpha}, t \in[0, T], 1 \leq i \leq n\right\} .
\end{aligned}
$$


From Theorem 1 and Theorem 4, we have

$$
\begin{aligned}
\mathcal{M} & \left\{\left[\min _{1 \leq i \leq n}\left(\frac{1}{T} \int_{0}^{T} S_{i t} d t\right)-K\right]^{+}\right. \\
& \left.\leq\left[\min _{1 \leq i \leq n}\left(\frac{1}{T} \int_{0}^{T} S_{i t}^{\alpha} d t\right)-K\right]^{+}\right\} \\
& \geq \mathcal{M}\left\{S_{i t} \leq S_{i t}^{\alpha}, t \in[0, T], 1 \leq i \leq n\right\} \\
& =\mathcal{M}\left\{\bigcap_{i=1}^{n}\left(S_{i t} \leq S_{i t}^{\alpha}\right), t \in[0, T]\right\} \\
& =\bigwedge_{i=1}^{n} \mathcal{M}\left\{S_{i t} \leq S_{i t}^{\alpha}, t \in[0, T]\right\} \\
& =\alpha
\end{aligned}
$$

and

$$
\begin{aligned}
\mathcal{M} & \left\{\left[\min _{1 \leq i \leq n}\left(\frac{1}{T} \int_{0}^{T} S_{i t} d t\right)-K\right]^{+}\right. \\
& \left.>\left[\min _{1 \leq i \leq n}\left(\frac{1}{T} \int_{0}^{T} S_{i t}^{\alpha} d t\right)-K\right]^{+}\right\} \\
& \geq \mathcal{M}\left\{S_{i t}>S_{i t}^{\alpha}, t \in[0, T], 1 \leq i \leq n\right\} \\
& =\mathcal{M}\left\{\bigcap_{i=1}^{n}\left(S_{i t}>S_{i t}^{\alpha}\right), t \in[0, T]\right\} \\
& =\bigwedge \mathfrak{N}\left\{S_{i t}>S_{i t}^{\alpha}, t \in[0, T]\right\} \\
& =1-\alpha .
\end{aligned}
$$

We can obtain

$$
\begin{aligned}
\mathcal{M} & \left\{\min _{1 \leq i \leq n}\left(\frac{1}{T} \int_{0}^{T} S_{i t} d t\right)-K\right]^{+} \\
\leq & {\left.\left[\min _{1 \leq i \leq n}\left(\frac{1}{T} \int_{0}^{T} S_{i t}^{\alpha} d t\right)-K\right]^{+}\right\} } \\
& +\mathcal{M}\left\{\left[\min _{1 \leq i \leq n}\left(\frac{1}{T} \int_{0}^{T} S_{i t} d t\right)-K\right]^{+}\right. \\
> & {\left.\left[\min _{1 \leq i \leq n}\left(\frac{1}{T} \int_{0}^{T} S_{i t}^{\alpha} d t\right)-K\right]^{+}\right\} } \\
= & 1
\end{aligned}
$$

by using duality axiom.
It follows from Eqs. (10)-(12) that

$$
\begin{aligned}
\mathcal{M} & \left\{\left[\min _{1 \leq i \leq n}\left(\frac{1}{T} \int_{0}^{T} S_{i t} d t\right)-K\right]^{+}\right. \\
& \left.\leq\left[\min _{1 \leq i \leq n}\left(\frac{1}{T} \int_{0}^{T} S_{i t}^{\alpha} d t\right)-K\right]^{+}\right\} \\
& =\alpha .
\end{aligned}
$$

Thus, the uncertain variable

$$
\left[\min _{1 \leq i \leq n}\left(\frac{1}{T} \int_{0}^{T} S_{i t} d t\right)-K\right]^{+}
$$

has an inverse uncertainty distribution

$$
\left[\min _{1 \leq i \leq n}\left(\frac{1}{T} \int_{0}^{T} S_{i t}^{\alpha} d t\right)-K\right]^{+}
$$

Hence, the pricing formula is

$$
\begin{aligned}
f_{2 c}^{a}= & \exp (-\gamma T) E\left[\left(\min _{1 \leq i \leq n}\left(\frac{1}{T} \int_{0}^{T} S_{i t} d t\right)-K\right)^{+}\right] \\
= & \exp (-\gamma T) \int_{0}^{1}\left[\min _{1 \leq i \leq n}\left(\frac{1}{T} \int_{0}^{T} S_{i t}^{\alpha} d t\right)-K\right]^{+} d \alpha \\
= & \exp (-\gamma T) \int_{0}^{1}\left[\operatorname { m i n } _ { 1 \leq i \leq n } \left(\frac{S_{i 0}}{T} \int_{0}^{T}\right.\right. \\
& \left.\left.\exp \left(\mu_{i} t+\frac{\sqrt{3} \sigma_{i} t}{\pi} \ln \frac{\alpha}{1-\alpha}\right) d t\right)-K\right]^{+} d \alpha
\end{aligned}
$$

The proof is completed.

Example 5 Consider that there are $S_{10}=12, S_{20}=11, K=$ $10, T=8$ for an arithmetic Asian rainbow call on min option with two underlying stocks. In Model (3), we assume that $\gamma=0.05, \mu_{1}=0.02, \sigma_{1}=0.04, \mu_{2}=0.01, \sigma_{2}=0.05$. Then, $f_{2 c}^{a}=1.1894$ by Theorem 11 .

Figure 5 demonstrates that the price $f_{2 c}^{a}$ is an increasing function of maturity time $T$ if other parameters are invariant.

\subsection{Geometric Asian rainbow call on min option}

Geometric Asian rainbow call on min option signifies that the holder can purchase min asset at the exercise price at the time of execution. The payoff of the option relies on the geometric mean of minimum asset price within an agreed period of time.

Assume that geometric Asian rainbow call on min option possesses a maturity time $T$ and an exercise price $K$ for Model (3). 


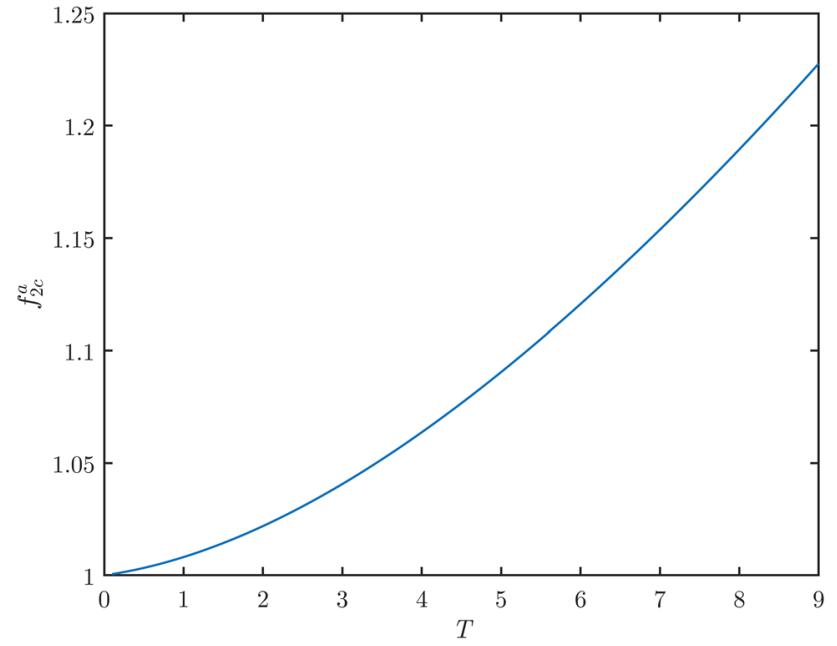

Fig. 5 Price $f_{2 c}^{a}$ concerning maturity time $T$ in Example 5

Firstly, from the holder's perspective, the payoff at time $T$ is

$\left[\min _{1 \leq i \leq n}\left(\exp \left(\frac{1}{T} \int_{0}^{T} \ln \left(S_{i t}\right) d t\right)\right)-K\right]^{+}$.

Let $f_{2 c}^{g}$ be the price of geometric Asian rainbow call on min option. Thus, at the initial moment, the holder of the option owns the net return

$-f_{2 c}^{g}+\exp (-\gamma T)\left[\min _{1 \leq i \leq n}\left(\exp \left(\frac{1}{T} \int_{0}^{T} \ln \left(S_{i t}\right) d t\right)\right)-K\right]^{+}$.

Secondly, from the seller's perspective, the payoff at time $T$ is

$-\left[\min _{1 \leq i \leq n}\left(\exp \left(\frac{1}{T} \int_{0}^{T} \ln \left(S_{i t}\right) d t\right)\right)-K\right]^{+}$.

Then, at the initial moment, the net return of the seller can be expressed:

$f_{2 c}^{g}-\exp (-\gamma T)\left[\min _{1 \leq i \leq n}\left(\exp \left(\frac{1}{T} \int_{0}^{T} \ln \left(S_{i t}\right) d t\right)\right)-K\right]^{+}$

Both should own uniform expected payoff by the fair price principle, that is

$$
\begin{aligned}
- & f_{2 c}^{g}+\exp (-\gamma T) E\left[\left(\min _{1 \leq i \leq n}\left(\exp \left(\frac{1}{T} \int_{0}^{T} \ln \left(S_{i t}\right) d t\right)\right)\right.\right. \\
& \left.-K)^{+}\right] \\
= & f_{2 c}^{g}-\exp (-\gamma T) E\left[\left(\min _{1 \leq i \leq n}\left(\exp \left(\frac{1}{T} \int_{0}^{T} \ln \left(S_{i t}\right) d t\right)\right)\right.\right. \\
& \left.-K)^{+}\right] .
\end{aligned}
$$

From the above, we conclude that the price of geometric Asian rainbow call on min option is equal to the present value of the expected payoff.

Definition 8 Suppose that a geometric Asian rainbow call on min option for Model (3) has a maturity time T and an exercise price $\mathrm{K}$. Then, its price is defined by

$$
f_{2 c}^{g}=\exp (-\gamma T) E\left[\left(\min _{1 \leq i \leq n}\left(\exp \left(\frac{1}{T} \int_{0}^{T} \ln \left(S_{i t}\right) d t\right)\right)-K\right)^{+}\right]
$$

Theorem 12 Suppose that a geometric Asian rainbow call on min option for Model (3) has a maturity time T and an exercise price $K$. Then, its price is

$$
\begin{aligned}
& f_{2 c}^{g}=\exp (-\gamma T) \int_{0}^{1}\left[\operatorname { m i n } _ { 1 \leq i \leq n } \left(S_{i 0}\right.\right. \\
& \left.\left.\quad \exp \left(\frac{\mu_{i} T}{2}+\frac{\sqrt{3} \sigma_{i} T}{2 \pi} \ln \frac{\alpha}{1-\alpha}\right)\right)-K\right]^{+} d \alpha .
\end{aligned}
$$

Proof We show that the uncertain variable

$$
\left[\min _{1 \leq i \leq n}\left(\exp \left(\frac{1}{T} \int_{0}^{T} \ln \left(S_{i t}\right) d t\right)\right)-K\right]^{+}
$$

has an inverse uncertainty distribution

$$
\left[\min _{1 \leq i \leq n}\left(\exp \left(\frac{1}{T} \int_{0}^{T} \ln \left(S_{i t}^{\alpha}\right) d t\right)\right)-K\right]^{+}
$$

where

$$
S_{i t}^{\alpha}=S_{i 0} \exp \left(\mu_{i} t+\frac{\sqrt{3} \sigma_{i} t}{\pi} \ln \frac{\alpha}{1-\alpha}\right) .
$$

Since $\exp \left(\int_{0}^{T} \ln \left(S_{t}\right) d t / T\right)$ is an increasing function with regard to $\ln (x)$, we derive

$$
\begin{aligned}
& \left\{\left[\min _{1 \leq i \leq n}\left(\exp \left(\frac{1}{T} \int_{0}^{T} \ln \left(S_{i t}\right) d t\right)\right)-K\right]^{+}\right. \\
& \left.\quad \leq\left[\min _{1 \leq i \leq n}\left(\exp \left(\frac{1}{T} \int_{0}^{T} \ln \left(S_{i t}^{\alpha}\right) d t\right)\right)-K\right]^{+}\right\} \\
& \quad \supseteq\left\{\min _{1 \leq i \leq n}\left(\exp \left(\frac{1}{T} \int_{0}^{T} \ln \left(S_{i t}\right) d t\right)\right)\right. \\
& \left.\quad \leq \min _{1 \leq i \leq n}\left(\exp \left(\frac{1}{T} \int_{0}^{T} \ln \left(S_{i t}^{\alpha}\right) d t\right)\right), t \in[0, T]\right\} \\
& \geq\left\{S_{i t} \leq S_{i t}^{\alpha}, t \in[0, T], 1 \leq i \leq n\right\}
\end{aligned}
$$


and

$$
\begin{aligned}
& \left\{\left[\min _{1 \leq i \leq n}\left(\exp \left(\frac{1}{T} \int_{0}^{T} \ln \left(S_{i t}\right) d t\right)\right)-K\right]^{+}\right. \\
& \left.\quad>\left[\min _{1 \leq i \leq n}\left(\exp \left(\frac{1}{T} \int_{0}^{T} \ln \left(S_{i t}^{\alpha}\right) d t\right)\right)-K\right]^{+}\right\} \\
& \quad \supseteq\left\{\min _{1 \leq i \leq n}\left(\exp \left(\frac{1}{T} \int_{0}^{T} \ln \left(S_{i t}\right) d t\right)\right)\right. \\
& \left.\quad>\min _{1 \leq i \leq n}\left(\exp \left(\frac{1}{T} \int_{0}^{T} \ln \left(S_{i t}^{\alpha}\right) d t\right)\right), t \in[0, T]\right\} \\
& \quad \supseteq\left\{S_{i t}>S_{i t}^{\alpha}, t \in[0, T], 1 \leq i \leq n\right\} .
\end{aligned}
$$

From Theorem 1 and Theorem 4, we have

$$
\begin{aligned}
\mathcal{M} & \left\{\left[\min _{1 \leq i \leq n}\left(\exp \left(\frac{1}{T} \int_{0}^{T} \ln \left(S_{i t}\right) d t\right)\right)-K\right]^{+}\right. \\
& \left.\leq\left[\min _{1 \leq i \leq n}\left(\exp \left(\frac{1}{T} \int_{0}^{T} \ln \left(S_{i t}^{\alpha}\right) d t\right)\right)-K\right]^{+}\right\} \\
& \geq \mathcal{M}\left\{S_{i t} \leq S_{i t}^{\alpha}, t \in[0, T], 1 \leq i \leq n\right\} \\
& =\mathcal{M}\left\{\bigcap_{i=1}^{n}\left(S_{i t} \leq S_{i t}^{\alpha}\right), t \in[0, T]\right\} \\
& =\bigwedge_{i=1}^{n} \mathcal{M}\left\{S_{i t} \leq S_{i t}^{\alpha}, t \in[0, T]\right\} \\
& =\alpha
\end{aligned}
$$

and

$$
\begin{aligned}
\mathcal{M} & \left\{\left[\min _{1 \leq i \leq n}\left(\exp \left(\frac{1}{T} \int_{0}^{T} \ln \left(S_{i t}\right) d t\right)\right)-K\right]^{+}\right. \\
& \left.>\left[\min _{1 \leq i \leq n}\left(\exp \left(\frac{1}{T} \int_{0}^{T} \ln \left(S_{i t}^{\alpha}\right) d t\right)\right)-K\right]^{+}\right\} \\
& \geq \mathcal{M}\left\{S_{i t}>S_{i t}^{\alpha}, t \in[0, T], 1 \leq i \leq n\right\} \\
& =\mathcal{M}\left\{\bigcap_{i=1}^{n}\left(S_{i t}>S_{i t}^{\alpha}\right), t \in[0, T]\right\} \\
& =\bigwedge_{i=1}^{n} \mathcal{M}\left\{S_{i t}>S_{i t}^{\alpha}, t \in[0, T]\right\} \\
& =1-\alpha .
\end{aligned}
$$

We can obtain

$$
\begin{aligned}
\mathcal{M} & \left\{\left[\min _{1 \leq i \leq n}\left(\exp \left(\frac{1}{T} \int_{0}^{T} \ln \left(S_{i t}\right) d t\right)\right)-K\right]^{+}\right. \\
\leq & {\left.\left[\min _{1 \leq i \leq n}\left(\exp \left(\frac{1}{T} \int_{0}^{T} \ln \left(S_{i t}^{\alpha}\right) d t\right)\right)-K\right]^{+}\right\} } \\
& +\mathcal{M}\left\{\left[\min _{1 \leq i \leq n}\left(\exp \left(\frac{1}{T} \int_{0}^{T} \ln \left(S_{i t}\right) d t\right)\right)-K\right]^{+}\right. \\
> & {\left.\left[\min _{1 \leq i \leq n}\left(\exp \left(\frac{1}{T} \int_{0}^{T} \ln \left(S_{i t}^{\alpha}\right) d t\right)\right)-K\right]^{+}\right\} } \\
= & 1
\end{aligned}
$$

by using duality axiom.

It follows from Eqs. (13)-(15) that

$$
\begin{aligned}
& \mathcal{M}\left\{\left[\min _{1 \leq i \leq n}\left(\exp \left(\frac{1}{T} \int_{0}^{T} \ln \left(S_{i t}\right) d t\right)\right)-K\right]^{+}\right. \\
& \left.\quad \leq\left[\min _{1 \leq i \leq n}\left(\exp \left(\frac{1}{T} \int_{0}^{T} \ln \left(S_{i t}^{\alpha}\right) d t\right)\right)-K\right]^{+}\right\}=\alpha .
\end{aligned}
$$

Thus, the uncertain variable

$\left[\min _{1 \leq i \leq n}\left(\exp \left(\frac{1}{T} \int_{0}^{T} \ln \left(S_{i t}\right) d t\right)\right)-K\right]^{+}$

has an inverse uncertainty distribution

$\left[\min _{1 \leq i \leq n}\left(\exp \left(\frac{1}{T} \int_{0}^{T} \ln \left(S_{i t}^{\alpha}\right) d t\right)\right)-K\right]^{+}$.

Hence, the pricing formula is

$$
\begin{aligned}
f_{2 c}^{g}= & \exp (-\gamma T) E\left[\left(\min _{1 \leq i \leq n}\left(\exp \left(\frac{1}{T} \int_{0}^{T} \ln \left(S_{i t}\right) d t\right)\right)-K\right)^{+}\right] \\
= & \exp (-\gamma T) \int_{0}^{1}\left[\min _{1 \leq i \leq n}\left(\exp \left(\frac{1}{T} \int_{0}^{T} \ln \left(S_{i t}^{\alpha}\right) d t\right)\right)-K\right]^{+} d \alpha \\
= & \exp (-\gamma T) \int_{0}^{1}\left[\min _{1 \leq i \leq n}\left(S_{i 0} \exp \left(\frac{\mu_{i} T}{2}+\frac{\sqrt{3} \sigma_{i} T}{2 \pi} \ln \frac{\alpha}{1-\alpha}\right)\right)\right. \\
& -K]^{+} d \alpha .
\end{aligned}
$$

The proof is completed.

Example 6 Consider that there are $S_{10}=12, S_{20}=11, K=$ $10, T=8$ for a geometric Asian rainbow call on min option with two underlying stocks. In Model (3), we assume that $\gamma=0.05, \mu_{1}=0.02, \sigma_{1}=0.04, \mu_{2}=0.01, \sigma_{2}=0.05$. Then, $f_{2 c}^{g}=1.1269$ by Theorem 12 .

Figure 6 demonstrates that the price $f_{2 c}^{g}$ is an increasing function of maturity time $T$ if other parameters are invariant. 


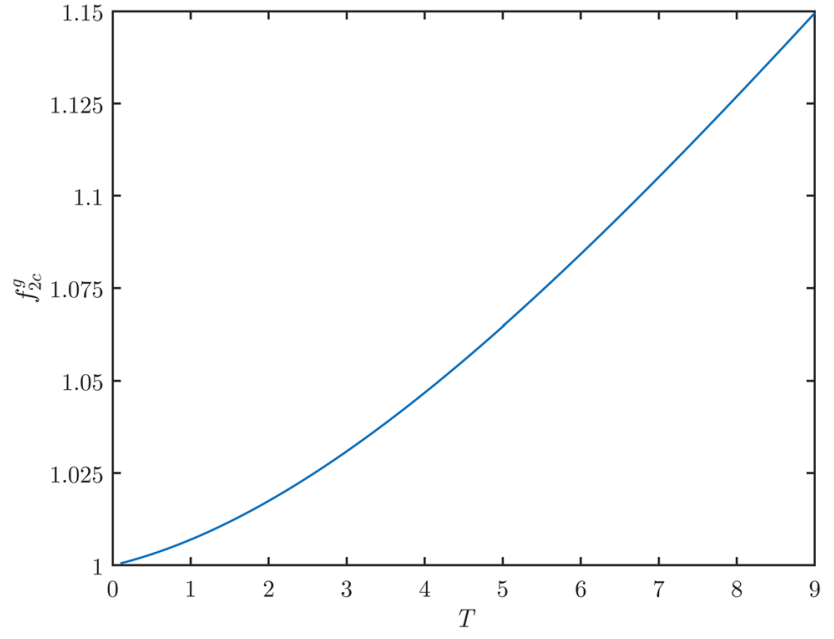

Fig. 6 Price $f_{2 c}^{g}$ concerning maturity time $T$ in Example 6

\section{Asian rainbow put option}

Asian rainbow put option is a contract that provides the holder with a right to sell the maximum or minimum asset at a given price at maturity time. The gain of the option depends on the mean of the maximum or minimum underlying asset price over its lifetime. The mean of underlying assets prices is calculated by the arithmetic average and geometric average methods. In this section, we study arithmetic Asian rainbow put on max option, geometric Asian rainbow put on max option, arithmetic Asian rainbow put on min option and geometric Asian rainbow put on min option. Furthermore, we obtain the pricing formulas of Asian rainbow put on max option and Asian rainbow put on min option by strict deduction.

\subsection{Arithmetic Asian rainbow put on max option}

Arithmetic Asian rainbow put on max option signifies that the holder can purchase maximum asset at the exercise price at the time of execution. The payoff of the option relies on the arithmetic mean of maximum asset price within an agreed period of time.

Assume that arithmetic Asian rainbow put on max option possesses a maturity time $T$ and an exercise price $K$ for Model (3).

Firstly, from the holder's perspective, the payoff at time $T$ is

$\left[K-\max _{1 \leq i \leq n}\left(\frac{1}{T} \int_{0}^{T} S_{i t} d t\right)\right]^{+}$.

Let $f_{1 p}^{a}$ be the price of arithmetic Asian rainbow put on max option. Thus, at the initial moment, the holder of the option owns the net return $-f_{1 p}^{a}+\exp (-\gamma T)\left[K-\max _{1 \leq i \leq n}\left(\frac{1}{T} \int_{0}^{T} S_{i t} d t\right)\right]^{+}$.

Secondly, from the seller's perspective, the payoff at time $T$ is

$-\left[K-\max _{1 \leq i \leq n}\left(\frac{1}{T} \int_{0}^{T} S_{i t} d t\right)\right]^{+}$

Then, at the initial moment, the net return of the seller can be expressed:

$f_{1 p}^{a}-\exp (-\gamma T)\left[K-\max _{1 \leq i \leq n}\left(\frac{1}{T} \int_{0}^{T} S_{i t} d t\right)\right]^{+}$.

Both should own uniform expected payoff by the fair price principle, that is

$$
\begin{aligned}
& -f_{1 p}^{a}+\exp (-\gamma T) E\left[\left(K-\max _{1 \leq i \leq n}\left(\frac{1}{T} \int_{0}^{T} S_{i t} d t\right)\right)^{+}\right] \\
& =f_{1 p}^{a}-\exp (-\gamma T) E\left[\left(K-\max _{1 \leq i \leq n}\left(\frac{1}{T} \int_{0}^{T} S_{i t} d t\right)\right)^{+}\right] .
\end{aligned}
$$

From the above, we conclude that the price of arithmetic Asian rainbow put on max option is equal to the present value of the expected payoff.

Definition 9 Suppose that an arithmetic Asian rainbow put on max option for Model (3) has a maturity time $T$ and an exercise price $K$. Then, its price is defined by

$f_{1 p}^{a}=\exp (-\gamma T) E\left[\left(K-\max _{1 \leq i \leq n}\left(\frac{1}{T} \int_{0}^{T} S_{i t} d t\right)\right)^{+}\right]$

Theorem 13 Suppose that an arithmetic Asian rainbow put on max option for Model (3) has a maturity time $T$ and an exercise price $K$. Then, its price is

$$
\begin{gathered}
f_{1 p}^{a}=\exp (-\gamma T) \int_{0}^{1}\left[K-\max _{1 \leq i \leq n}\left(\frac{S_{i 0}}{T} \int_{0}^{T}\right.\right. \\
\left.\left.\exp \left(\mu_{i} t+\frac{\sqrt{3} \sigma_{i} t}{\pi} \ln \frac{\alpha}{1-\alpha}\right) d t\right)\right]^{+} d \alpha
\end{gathered}
$$

Proof We show that the uncertain variable

$$
\left[K-\max _{1 \leq i \leq n}\left(\frac{1}{T} \int_{0}^{T} S_{i t} d t\right)\right]^{+}
$$

has an inverse uncertainty distribution

$$
\left[K-\max _{1 \leq i \leq n}\left(\frac{1}{T} \int_{0}^{T} S_{i t}^{1-\alpha} d t\right)\right]^{+}
$$


Asian rainbow option pricing formulas of uncertain stock model

8865

where

$$
S_{i t}^{1-\alpha}=S_{i 0} \exp \left(\mu_{i} t+\frac{\sqrt{3} \sigma_{i} t}{\pi} \ln \frac{1-\alpha}{\alpha}\right) .
$$

Since $\int_{0}^{T} x d t / T$ is an increasing function with regard to $x$, we derive

$$
\begin{aligned}
& \left\{\left[K-\max _{1 \leq i \leq n}\left(\frac{1}{T} \int_{0}^{T} S_{i t} d t\right)\right]^{+}\right. \\
& \left.\quad \leq\left[K-\max _{1 \leq i \leq n}\left(\frac{1}{T} \int_{0}^{T} S_{i t}^{1-\alpha} d t\right)\right]^{+}\right\} \\
& \quad \supseteq\left\{\max _{1 \leq i \leq n}\left(\frac{1}{T} \int_{0}^{T} S_{i t} d t\right)\right. \\
& \left.\quad \geq \max _{1 \leq i \leq n}\left(\frac{1}{T} \int_{0}^{T} S_{i t}^{1-\alpha} d t\right), t \in[0, T]\right\} \\
& \quad \supseteq\left\{S_{i t} \geq S_{i t}^{1-\alpha}, t \in[0, T], 1 \leq i \leq n\right\}
\end{aligned}
$$

and

$$
\begin{aligned}
& \left\{\left[K-\max _{1 \leq i \leq n}\left(\frac{1}{T} \int_{0}^{T} S_{i t} d t\right)\right]^{+}\right. \\
& \left.\quad>\left[K-\max _{1 \leq i \leq n}\left(\frac{1}{T} \int_{0}^{T} S_{i t}^{1-\alpha} d t\right)\right]^{+}\right\} \\
& \quad \supseteq\left\{\max _{1 \leq i \leq n}\left(\frac{1}{T} \int_{0}^{T} S_{i t} d t\right)\right. \\
& \left.\quad<\max _{1 \leq i \leq n}\left(\frac{1}{T} \int_{0}^{T} S_{i t}^{1-\alpha} d t\right), t \in[0, T]\right\} \\
& \quad \supseteq\left\{S_{i t}<S_{i t}^{1-\alpha}, t \in[0, T], 1 \leq i \leq n\right\} .
\end{aligned}
$$

From Theorem 1 and Theorem 4, we have

$$
\begin{aligned}
\mathcal{M} & \left\{\left[K-\max _{1 \leq i \leq n}\left(\frac{1}{T} \int_{0}^{T} S_{i t} d t\right)\right]^{+}\right. \\
& \left.\leq\left[K-\max _{1 \leq i \leq n}\left(\frac{1}{T} \int_{0}^{T} S_{i t}^{1-\alpha} d t\right)\right]^{+}\right\} \\
& \geq \mathcal{M}\left\{S_{i t} \geq S_{i t}^{1-\alpha}, t \in[0, T], 1 \leq i \leq n\right\} \\
& =\mathcal{M}\left\{\bigcap_{i=1}^{n}\left(S_{i t} \geq S_{i t}^{1-\alpha}\right), t \in[0, T]\right\} \\
& =\bigwedge \mathcal{N}\left\{S_{i t} \geq S_{i t}^{1-\alpha}, t \in[0, T]\right\} \\
& =\alpha
\end{aligned}
$$

and

$$
\begin{aligned}
\mathcal{M} & \left\{\left[K-\max _{1 \leq i \leq n}\left(\frac{1}{T} \int_{0}^{T} S_{i t} d t\right)\right]^{+}\right. \\
& \left.>\left[K-\max _{1 \leq i \leq n}\left(\frac{1}{T} \int_{0}^{T} S_{i t}^{1-\alpha} d t\right)\right]^{+}\right\} \\
\geq & \mathcal{M}\left\{S_{i t}<S_{i t}^{1-\alpha}, t \in[0, T], 1 \leq i \leq n\right\} \\
& =\mathcal{M}\left\{\bigcap_{i=1}^{n}\left(S_{i t}<S_{i t}^{1-\alpha}\right), t \in[0, T]\right\} \\
& =\bigwedge_{i=1}^{n} \mathcal{M}\left\{S_{i t}<S_{i t}^{1-\alpha}, t \in[0, T]\right\} \\
& =1-\alpha .
\end{aligned}
$$

We can obtain

$$
\begin{aligned}
\mathcal{M} & \left\{\left[K-\max _{1 \leq i \leq n}\left(\frac{1}{T} \int_{0}^{T} S_{i t} d t\right)\right]^{+}\right. \\
\leq & {\left.\left[K-\max _{1 \leq i \leq n}\left(\frac{1}{T} \int_{0}^{T} S_{i t}^{1-\alpha} d t\right)\right]^{+}\right\} } \\
& +\mathcal{M}\left\{\left[K-\max _{1 \leq i \leq n}\left(\frac{1}{T} \int_{0}^{T} S_{i t} d t\right)\right]^{+}\right. \\
> & {\left.\left[K-\max _{1 \leq i \leq n}\left(\frac{1}{T} \int_{0}^{T} S_{i t}^{1-\alpha} d t\right)\right]^{+}\right\} } \\
= & 1
\end{aligned}
$$

by using duality axiom.

It follows from Eqs. (16)-(18) that

$$
\begin{aligned}
\mathcal{M} & \left\{\left[K-\max _{1 \leq i \leq n}\left(\frac{1}{T} \int_{0}^{T} S_{i t} d t\right)\right]^{+}\right. \\
& \left.\leq\left[K-\max _{1 \leq i \leq n}\left(\frac{1}{T} \int_{0}^{T} S_{i t}^{1-\alpha} d t\right)\right]^{+}\right\} \\
& =\alpha .
\end{aligned}
$$

Thus, the uncertain variable

$$
\left[K-\max _{1 \leq i \leq n}\left(\frac{1}{T} \int_{0}^{T} S_{i t} d t\right)\right]^{+}
$$

has an inverse uncertainty distribution

$$
\left[K-\max _{1 \leq i \leq n}\left(\frac{1}{T} \int_{0}^{T} S_{i t}^{1-\alpha} d t\right)\right]^{+}
$$

Springer 
Hence, the pricing formula is

$$
\begin{aligned}
f_{1 p}^{a}= & \exp (-\gamma T) E\left[\left(K-\max _{1 \leq i \leq n}\left(\frac{1}{T} \int_{0}^{T} S_{i t} d t\right)\right)^{+}\right] \\
= & \exp (-\gamma T) \int_{0}^{1}\left[K-\max _{1 \leq i \leq n}\left(\frac{1}{T} \int_{0}^{T} S_{i t}^{1-\alpha} d t\right)\right]^{+} d \alpha \\
= & \exp (-\gamma T) \int_{0}^{1}\left[K-\max _{1 \leq i \leq n}\left(\frac{S_{i 0}}{T} \int_{0}^{T}\right.\right. \\
& \left.\left.\exp \left(\mu_{i} t+\frac{\sqrt{3} \sigma_{i} t}{\pi} \ln \frac{1-\alpha}{\alpha}\right) d t\right)\right]^{+} d \alpha \\
= & \exp (-\gamma T) \int_{0}^{1}\left[K-\max _{1 \leq i \leq n}\left(\frac{S_{i 0}}{T} \int_{0}^{T}\right.\right. \\
& \left.\left.\exp \left(\mu_{i} t+\frac{\sqrt{3} \sigma_{i} t}{\pi} \ln \frac{\alpha}{1-\alpha}\right) d t\right)\right]^{+} d \alpha .
\end{aligned}
$$

The proof is completed.

Example 7 Consider that there are $S_{10}=9, S_{20}=8, K=$ $10, T=6$ for an arithmetic Asian rainbow put on max option with two underlying stocks. In Model (3), we assume that $\gamma=0.02, \mu_{1}=-0.02, \sigma_{1}=0.05, \mu_{2}=-0.03, \sigma_{2}=0.06$. Then, $f_{1 p}^{a}=1.2357$ by Theorem 13 .

Figure 7 demonstrates that the price $f_{1 p}^{a}$ is an increasing function of maturity time $T$ if other parameters are invariant.

\subsection{Geometric Asian rainbow put on max option}

Geometric Asian rainbow put on max option signifies that the holder can sell maximum asset at the exercise price at the time of execution. The payoff of the option relies on the geometric mean of maximum asset price within an agreed period of time.

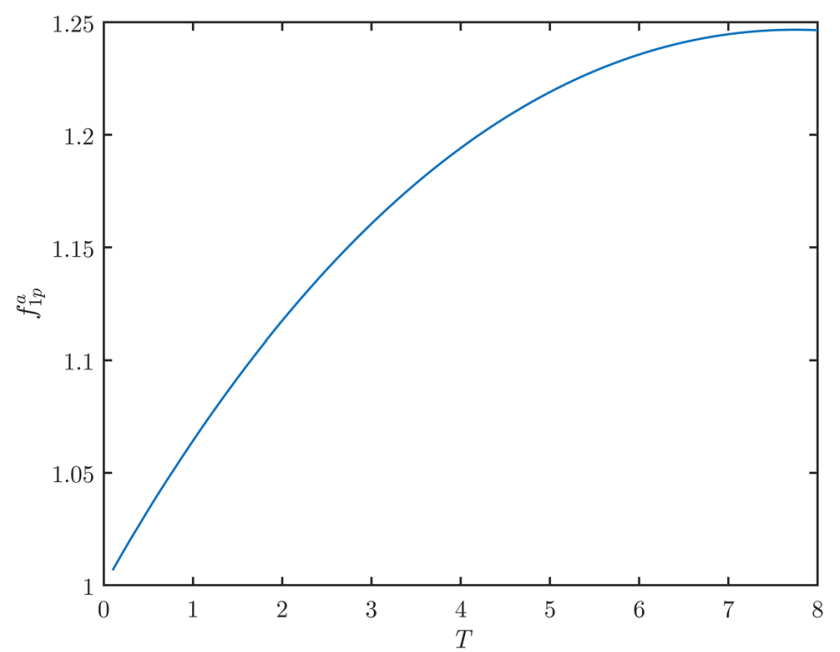

Fig. 7 Price $f_{1 p}^{a}$ concerning maturity time $T$ in Example 7
Assume that geometric Asian rainbow put on max option possesses a maturity time $T$ and an exercise price $K$ for Model (3).

Firstly, from the holder's perspective, the payoff at time $T$ is

$$
\left[K-\max _{1 \leq i \leq n}\left(\exp \left(\frac{1}{T} \int_{0}^{T} \ln \left(S_{i t}\right) d t\right)\right)\right]^{+}
$$

Let $f_{1 p}^{g}$ be the price of geometric Asian rainbow put on max option. Thus, at the initial moment, the holder of the option owns the net return

$-f_{1 p}^{g}+\exp (-\gamma T)\left[K-\max _{1 \leq i \leq n}\left(\exp \left(\frac{1}{T} \int_{0}^{T} \ln \left(S_{i t}\right) d t\right)\right)\right]^{+}$.

Secondly, from the seller's perspective, the payoff at time $T$ is

$-\left[K-\max _{1 \leq i \leq n}\left(\exp \left(\frac{1}{T} \int_{0}^{T} \ln \left(S_{i t}\right) d t\right)\right)\right]^{+}$

Then, at the initial moment, the net return of the seller can be expressed:

$f_{1 p}^{g}-\exp (-\gamma T)\left[K-\max _{1 \leq i \leq n}\left(\exp \left(\frac{1}{T} \int_{0}^{T} \ln \left(S_{i t}\right) d t\right)\right)\right]^{+}$

Both should own uniform expected payoff by the fair price principle, that is

$$
\begin{aligned}
& -f_{1 p}^{g}+\exp (-\gamma T) E[(K \\
& \left.\left.-\max _{1 \leq i \leq n}\left(\exp \left(\frac{1}{T} \int_{0}^{T} \ln \left(S_{i t}\right) d t\right)\right)\right)^{+}\right] \\
& =f_{1 p}^{g}-\exp (-\gamma T) E[(K \\
& \left.\left.\quad-\max _{1 \leq i \leq n}\left(\exp \left(\frac{1}{T} \int_{0}^{T} \ln \left(S_{i t}\right) d t\right)\right)\right)^{+}\right] .
\end{aligned}
$$

From the above, we conclude that the price of geometric Asian rainbow put on max option is equal to the present value of the expected payoff.

Definition 10 Suppose that a geometric Asian rainbow put on max option for Model (3) has a maturity time $\mathrm{T}$ and an exercise price $\mathrm{K}$. Then, its price is defined by

$$
\begin{aligned}
f_{1 p}^{g} & =\exp (-\gamma T) E[(K \\
& \left.\left.-\max _{1 \leq i \leq n}\left(\exp \left(\frac{1}{T} \int_{0}^{T} \ln \left(S_{i t}\right) d t\right)\right)\right)^{+}\right] .
\end{aligned}
$$


Theorem 14 Suppose that a geometric Asian rainbow put on max option for Model (3) has a maturity time $T$ and an exercise price $K$. Then, its price is

$$
\begin{aligned}
& f_{1 p}^{g}=\exp (-\gamma T) \int_{0}^{1}\left[K-\max _{1 \leq i \leq n}\left(S_{i 0}\right.\right. \\
& \left.\left.\quad \exp \left(\frac{\mu_{i} T}{2}+\frac{\sqrt{3} \sigma_{i} T}{2 \pi} \ln \frac{\alpha}{1-\alpha}\right)\right)\right]^{+} d \alpha .
\end{aligned}
$$

Proof We show that the uncertain variable

$$
\left[K-\max _{1 \leq i \leq n}\left(\exp \left(\frac{1}{T} \int_{0}^{T} \ln \left(S_{i t}\right) d t\right)\right)\right]^{+}
$$

has an inverse uncertainty distribution

$$
\left[K-\max _{1 \leq i \leq n}\left(\exp \left(\frac{1}{T} \int_{0}^{T} \ln \left(S_{i t}^{1-\alpha}\right) d t\right)\right)\right]^{+}
$$

where

$$
S_{i t}^{1-\alpha}=S_{i 0} \exp \left(\mu_{i} t+\frac{\sqrt{3} \sigma_{i} t}{\pi} \ln \frac{1-\alpha}{\alpha}\right) .
$$

Since $\exp \left(\int_{0}^{T} \ln (x) d t / T\right)$ is an increasing function with regard to $\ln (x)$, we derive

$$
\begin{aligned}
& \left\{\left[K-\max _{1 \leq i \leq n}\left(\exp \left(\frac{1}{T} \int_{0}^{T} \ln \left(S_{i t}\right) d t\right)\right)\right]^{+}\right. \\
& \left.\quad \leq\left[K-\max _{1 \leq i \leq n}\left(\exp \left(\frac{1}{T} \int_{0}^{T} \ln \left(S_{i t}^{1-\alpha}\right) d t\right)\right)\right]^{+}\right\} \\
& \quad \supseteq\left\{\max _{1 \leq i \leq n}\left(\exp \left(\frac{1}{T} \int_{0}^{T} \ln \left(S_{i t}\right) d t\right)\right)\right. \\
& \left.\quad \geq \max _{1 \leq i \leq n}\left(\exp \left(\frac{1}{T} \int_{0}^{T} \ln \left(S_{i t}^{1-\alpha}\right) d t\right)\right), t \in[0, T]\right\} \\
& \quad \supseteq\left\{S_{i t} \geq S_{i t}^{1-\alpha}, t \in[0, T], 1 \leq i \leq n\right\}
\end{aligned}
$$

and

$$
\begin{aligned}
& \left\{\left[K-\max _{1 \leq i \leq n}\left(\exp \left(\frac{1}{T} ! \int_{0}^{T} \ln \left(S_{i t}\right) d t\right)\right)\right]^{+}\right. \\
& \left.\quad>\left[K-\max _{1 \leq i \leq n}\left(\exp \left(\frac{1}{T} \int_{0}^{T} \ln \left(S_{i t}^{1-\alpha}\right) d t\right)\right)\right]^{+}\right\} \\
& \quad \supseteq\left\{\max _{1 \leq i \leq n}\left(\exp \left(\frac{1}{T} \int_{0}^{T} \ln \left(S_{i t}\right) d t\right)\right)\right. \\
& \left.\quad<\max _{1 \leq i \leq n}\left(\exp \left(\frac{1}{T} \int_{0}^{T} \ln \left(S_{i t}^{1-\alpha}\right) d t\right)\right), t \in[0, T]\right\} \\
& \quad \supseteq\left\{S_{i t}<S_{i t}^{1-\alpha}, t \in[0, T], 1 \leq i \leq n\right\} .
\end{aligned}
$$

From Theorem 1 and Theorem 4, we have

$$
\begin{aligned}
\mathcal{M} & \left\{\left[K-\max _{1 \leq i \leq n}\left(\exp \left(\frac{1}{T} \int_{0}^{T} \ln \left(S_{i t}\right) d t\right)\right)\right]^{+}\right. \\
& \left.\leq\left[K-\max _{1 \leq i \leq n}\left(\exp \left(\frac{1}{T} \int_{0}^{T} \ln \left(S_{i t}^{1-\alpha}\right) d t\right)\right)\right]^{+}\right\} \\
& \geq \mathcal{N}\left\{S_{i t} \geq S_{i t}^{1-\alpha}, t \in[0, T], 1 \leq i \leq n\right\} \\
& =\mathcal{M}\left\{\bigcap_{i=1}^{n}\left(S_{i t} \geq S_{i t}^{1-\alpha}\right), t \in[0, T]\right\} \\
& =\bigwedge_{i=1} \mathcal{M}\left\{S_{i t} \geq S_{i t}^{1-\alpha}, t \in[0, T]\right\} \\
& =\alpha
\end{aligned}
$$

and

$$
\begin{aligned}
\mathcal{M} & \left\{\left[K-\max _{1 \leq i \leq n}\left(\exp \left(\frac{1}{T} \int_{0}^{T} \ln \left(S_{i t}\right) d t\right)\right)\right]^{+}\right. \\
& \left.>\left[K-\max _{1 \leq i \leq n}\left(\exp \left(\frac{1}{T} \int_{0}^{T} \ln \left(S_{i t}^{1-\alpha}\right) d t\right)\right)\right]^{+}\right\} \\
\geq & \mathcal{M}\left\{S_{i t}<S_{i t}^{1-\alpha}, t \in[0, T], 1 \leq i \leq n\right\} \\
& =\mathcal{M}\left\{\bigcap_{i=1}^{n}\left(S_{i t}<S_{i t}^{1-\alpha}\right), t \in[0, T]\right\} \\
& =\bigwedge_{i=1} \mathcal{M}\left\{S_{i t}<S_{i t}^{1-\alpha}, t \in[0, T]\right\} \\
& =1-\alpha .
\end{aligned}
$$

We can obtain

$$
\begin{aligned}
\mathcal{M} & \left\{\left[K-\max _{1 \leq i \leq n}\left(\exp \left(\frac{1}{T} \int_{0}^{T} \ln \left(S_{i t}\right) d t\right)\right)\right]^{+}\right. \\
\leq & {\left.\left[K-\max _{1 \leq i \leq n}\left(\exp \left(\frac{1}{T} \int_{0}^{T} \ln \left(S_{i t}^{1-\alpha}\right) d t\right)\right)\right]^{+}\right\} } \\
& +\mathcal{M}\left\{\left[K-\max _{1 \leq i \leq n}\left(\exp \left(\frac{1}{T} \int_{0}^{T} \ln \left(S_{i t}\right) d t\right)\right)\right]^{+}\right. \\
> & {\left.\left[K-\max _{1 \leq i \leq n}\left(\exp \left(\frac{1}{T} \int_{0}^{T} \ln \left(S_{i t}^{1-\alpha}\right) d t\right)\right)\right]^{+}\right\} } \\
= & 1
\end{aligned}
$$

by using duality axiom. 
It follows from Eqs. (19)-(21) that

$$
\begin{aligned}
& \mathcal{M}\left\{\left[K-\max _{1 \leq i \leq n}\left(\exp \left(\frac{1}{T} \int_{0}^{T} \ln \left(S_{i t}\right) d t\right)\right)\right]^{+}\right. \\
& \left.\quad \leq\left[K-\max _{1 \leq i \leq n}\left(\exp \left(\frac{1}{T} \int_{0}^{T} \ln \left(S_{i t}^{1-\alpha}\right) d t\right)\right)\right]^{+}\right\}=\alpha .
\end{aligned}
$$

Thus, the uncertain variable

$$
\left[K-\max _{1 \leq i \leq n}\left(\exp \left(\frac{1}{T} \int_{0}^{T} \ln \left(S_{i t}\right) d t\right)\right)\right]^{+}
$$

has an inverse uncertainty distribution

$$
\left[K-\max _{1 \leq i \leq n}\left(\exp \left(\frac{1}{T} \int_{0}^{T} \ln \left(S_{i t}^{1-\alpha}\right) d t\right)\right)\right]^{+} .
$$

Hence, the pricing formula is

$$
\begin{aligned}
f_{1 p}^{g}= & \exp (-\gamma T) E[(K \\
& \left.\left.-\max _{1 \leq i \leq n}\left(\exp \left(\frac{1}{T} \int_{0}^{T} \ln \left(S_{i t}\right) d t\right)\right)\right)^{+}\right] \\
= & \exp (-\gamma T) \int_{0}^{1}[K \\
& \left.-\max _{1 \leq i \leq n}\left(\exp \left(\frac{1}{T} \int_{0}^{T} \ln \left(S_{i t}^{1-\alpha}\right) d t\right)\right)\right]^{+} d \alpha \\
= & \exp (-\gamma T) \int_{0}^{1}[K \\
& \left.-\max _{1 \leq i \leq n}\left(S_{i 0} \exp \left(\frac{\mu_{i} T}{2}+\frac{\sqrt{3} \sigma_{i} T}{2 \pi} \ln \frac{1-\alpha}{\alpha}\right)\right)\right]^{+} d \alpha \\
= & \exp (-\gamma T) \int_{0}^{1}[K \\
& \left.-\max _{1 \leq i \leq n}\left(S_{i 0} \exp \left(\frac{\mu_{i} T}{2}+\frac{\sqrt{3} \sigma_{i} T}{2 \pi} \ln \frac{\alpha}{1-\alpha}\right)\right)\right]^{+} d \alpha .
\end{aligned}
$$

The proof is completed.

Example 8 Consider that there are $S_{10}=9, S_{20}=8, K=$ $10, T=6$ for a geometric Asian rainbow put on max option with two underlying stocks. In Model (3), we assume that $\gamma=0.02, \mu_{1}=-0.02, \sigma_{1}=0.05, \mu_{2}=-0.03, \sigma_{2}=0.06$. Then, $f_{1 p}^{g}=1.2665$ by Theorem 14 .

Figure 8 demonstrates that the price $f_{1 p}^{g}$ is an increasing function of maturity time $T$ if other parameters are invariant.

\subsection{Arithmetic Asian rainbow put on min option}

Arithmetic Asian rainbow put on min option signifies that the holder can sell minimum asset at the exercise price at

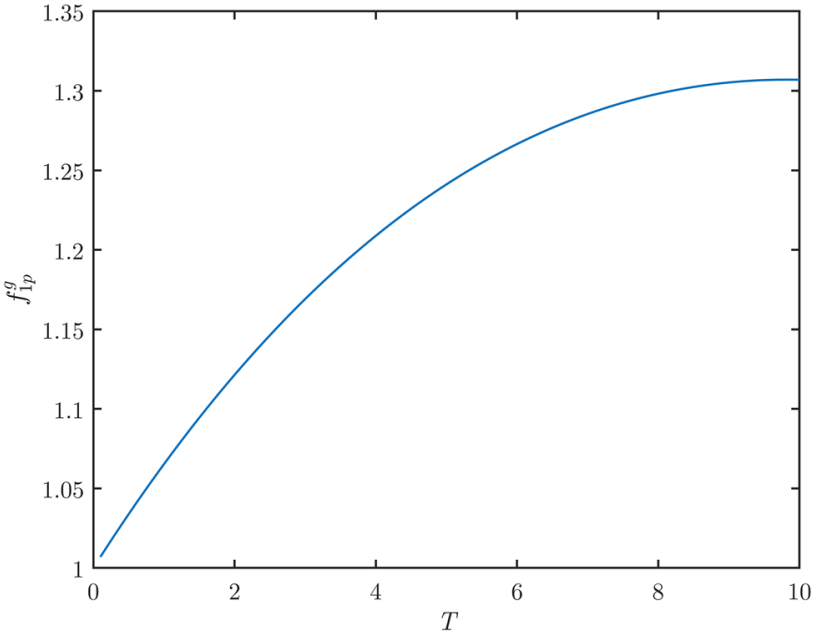

Fig. 8 Price $f_{1 p}^{g}$ concerning maturity time $T$ in Example 8

the time of execution. The payoff of the option relies on the arithmetic mean of minimum asset price within an agreed period of time.

Assume that arithmetic Asian rainbow put on min option possesses a maturity time $T$ and an exercise price $K$ for Model (3).

Firstly, from the holder's perspective, the payoff at time $T$ is

$\left[K-\min _{1 \leq i \leq n}\left(\frac{1}{T} \int_{0}^{T} S_{i t} d t\right)\right]^{+}$

Let $f_{2 p}^{a}$ be the price of arithmetic Asian rainbow put on min option. Thus, at the initial moment, the holder of the option owns the net return

$-f_{2 p}^{a}+\exp (-\gamma T)\left[K-\min _{1 \leq i \leq n}\left(\frac{1}{T} \int_{0}^{T} S_{i t} d t\right)\right]^{+}$

Secondly, from the seller's perspective, the payoff at time $T$ is

$-\left[K-\min _{1 \leq i \leq n}\left(\frac{1}{T} \int_{0}^{T} S_{i t} d t\right)\right]^{+}$.

Then, at the initial moment, the net return of the seller can be expressed:

$f_{2 p}^{a}-\exp (-\gamma T)\left[K-\min _{1 \leq i \leq n}\left(\frac{1}{T} \int_{0}^{T} S_{i t} d t\right)\right]^{+}$. 
Both should own uniform expected payoff by the fair price principle, that is

$$
\begin{aligned}
& -f_{2 p}^{a}+\exp (-\gamma T) E\left[\left(K-\min _{1 \leq i \leq n}\left(\frac{1}{T} \int_{0}^{T} S_{i t} d t\right)\right)^{+}\right] \\
& =f_{2 p}^{a}-\exp (-\gamma T) E\left[\left(K-\min _{1 \leq i \leq n}\left(\frac{1}{T} \int_{0}^{T} S_{i t} d t\right)\right)^{+}\right] .
\end{aligned}
$$

From the above, we conclude that the price of arithmetic Asian rainbow put on min option is equal to the present value of the expected payoff.

Definition 11 Suppose that an arithmetic Asian rainbow put on min option for Model (3) has a maturity time $T$ and an exercise price $K$. Then, its price is defined by

$$
f_{2 p}^{a}=\exp (-\gamma T) E\left[\left(K-\min _{1 \leq i \leq n}\left(\frac{1}{T} \int_{0}^{T} S_{i t} d t\right)\right)^{+}\right] .
$$

Theorem 15 Suppose that an arithmetic Asian rainbow put on min option for Model (3) has a maturity time $T$ and an exercise price $K$. Then, its price is

$$
\begin{gathered}
f_{2 p}^{a}=\exp (-\gamma T) \int_{0}^{1}\left[K-\min _{1 \leq i \leq n}\left(\frac{S_{i 0}}{T} \int_{0}^{T}\right.\right. \\
\left.\left.\exp \left(\mu_{i} t+\frac{\sqrt{3} \sigma_{i} t}{\pi} \ln \frac{\alpha}{1-\alpha}\right) d t\right)\right]^{+} d \alpha .
\end{gathered}
$$

Proof We show that the uncertain variable

$$
\left[K-\min _{1 \leq i \leq n}\left(\frac{1}{T} \int_{0}^{T} S_{i t} d t\right)\right]^{+}
$$

has an inverse uncertainty distribution

$$
\left[K-\min _{1 \leq i \leq n}\left(\frac{1}{T} \int_{0}^{T} S_{i t}^{1-\alpha} d t\right)\right]^{+}
$$

where

$$
S_{i t}^{1-\alpha}=S_{i 0} \exp \left(\mu_{i} t+\frac{\sqrt{3} \sigma_{i} t}{\pi} \ln \frac{1-\alpha}{\alpha}\right) .
$$

Since $\int_{0}^{T} x d t / T$ is an increasing function with regard to $x$, we derive

$$
\begin{aligned}
& \left\{\left[K-\min _{1 \leq i \leq n}\left(\frac{1}{T} \int_{0}^{T} S_{i t} d t\right)\right]^{+}\right. \\
& \left.\quad \leq\left[K-\min _{1 \leq i \leq n}\left(\frac{1}{T} \int_{0}^{T} S_{i t}^{1-\alpha} d t\right)\right]^{+}\right\} \\
& \geq\left\{\min _{1 \leq i \leq n}\left(\frac{1}{T} \int_{0}^{T} S_{i t} d t\right) \geq \min _{1 \leq i \leq n}\left(\frac{1}{T} \int_{0}^{T} S_{i t}^{1-\alpha} d t\right), t \in[0, T]\right\} \\
& \geq\left\{S_{i t} \geq S_{i t}^{1-\alpha}, t \in[0, T], 1 \leq i \leq n\right\}
\end{aligned}
$$

and

$$
\begin{aligned}
& \left\{\left[K-\min _{1 \leq i \leq n}\left(\frac{1}{T} \int_{0}^{T} S_{i t} d t\right)\right]^{+}\right. \\
& \left.\quad>\left[K-\min _{1 \leq i \leq n}\left(\frac{1}{T} \int_{0}^{T} S_{i t}^{1-\alpha} d t\right)\right]^{+}\right\} \\
& \quad \supseteq\left\{\min _{1 \leq i \leq n}\left(\frac{1}{T} \int_{0}^{T} S_{i t} d t\right)\right. \\
& \left.\quad<\min _{1 \leq i \leq n}\left(\frac{1}{T} \int_{0}^{T} S_{i t}^{1-\alpha} d t\right), t \in[0, T]\right\} \\
& \geq\left\{S_{i t}<S_{i t}^{1-\alpha}, t \in[0, T], 1 \leq i \leq n\right\} .
\end{aligned}
$$

From Theorem 1 and Theorem 4, we have

$$
\begin{aligned}
\mathcal{M} & \left\{\left[K-\min _{1 \leq i \leq n}\left(\frac{1}{T} \int_{0}^{T} S_{i t} d t\right)\right]^{+}\right. \\
& \left.\leq\left[K-\min _{1 \leq i \leq n}\left(\frac{1}{T} \int_{0}^{T} S_{i t}^{1-\alpha} d t\right)\right]^{+}\right\} \\
& \geq \mathcal{M}\left\{S_{i t} \geq S_{i t}^{1-\alpha}, t \in[0, T], 1 \leq i \leq n\right\} \\
& =\mathcal{M}\left\{\bigcap_{i=1}^{n}\left(S_{i t} \geq S_{i t}^{1-\alpha}\right), t \in[0, T]\right\} \\
& =\bigwedge_{i=1}^{n} \mathcal{M}\left\{S_{i t} \geq S_{i t}^{1-\alpha}, t \in[0, T]\right\} \\
& =\alpha
\end{aligned}
$$


and

$$
\begin{aligned}
\mathcal{M} & \left\{\left[K-\min _{1 \leq i \leq n}\left(\frac{1}{T} \int_{0}^{T} S_{i t} d t\right)\right]^{+}\right. \\
& \left.>\left[K-\min _{1 \leq i \leq n}\left(\frac{1}{T} \int_{0}^{T} S_{i t}^{\alpha} d t\right)\right]^{+}\right\} \\
& \geq \mathcal{M}\left\{S_{i t}<S_{i t}^{1-\alpha}, t \in[0, T], 1 \leq i \leq n\right\} \\
& =\mathcal{M}\left\{\bigcap_{i=1}^{n}\left(S_{i t}<S_{i t}^{1-\alpha}\right), t \in[0, T]\right\} \\
& =\bigwedge_{i=1}^{n} \mathcal{N}\left\{S_{i t}<S_{i t}^{1-\alpha}, t \in[0, T]\right\} \\
& =1-\alpha .
\end{aligned}
$$

We can obtain

$$
\begin{aligned}
\mathcal{M} & \left\{\left[K-\min _{1 \leq i \leq n}\left(\frac{1}{T} \int_{0}^{T} S_{i t} d t\right)\right]^{+}\right. \\
\leq & {\left.\left[K-\min _{1 \leq i \leq n}\left(\frac{1}{T} \int_{0}^{T} S_{i t}^{1-\alpha} d t\right)\right]^{+}\right\} } \\
& +\mathcal{M}\left\{\left[K-\min _{1 \leq i \leq n}\left(\frac{1}{T} \int_{0}^{T} S_{i t} d t\right)\right]^{+}\right. \\
> & {\left.\left[K-\min _{1 \leq i \leq n}\left(\frac{1}{T} \int_{0}^{T} S_{i t}^{1-\alpha} d t\right)\right]^{+}\right\} } \\
= & 1
\end{aligned}
$$

by using duality axiom.

It follows from Eqs. (22)-(24) that

$$
\begin{aligned}
& \mathcal{M}\left\{\left[K-\min _{1 \leq i \leq n}\left(\frac{1}{T} \int_{0}^{T} S_{i t} d t\right)\right]^{+}\right. \\
& \left.\quad \leq\left[K-\min _{1 \leq i \leq n}\left(\frac{1}{T} \int_{0}^{T} S_{i t}^{1-\alpha} d t\right)\right]^{+}\right\} \\
& \quad=\alpha .
\end{aligned}
$$

Thus, the uncertain variable

$$
\left[K-\min _{1 \leq i \leq n}\left(\frac{1}{T} \int_{0}^{T} S_{i t} d t\right)\right]^{+}
$$

has an inverse uncertainty distribution

$$
\left[K-\min _{1 \leq i \leq n}\left(\frac{1}{T} \int_{0}^{T} S_{i t}^{1-\alpha} d t\right)\right]^{+} .
$$

Hence, the pricing formula is

$$
\begin{aligned}
f_{2 p}^{a}= & \exp (-\gamma T) E\left[\left(K-\min _{1 \leq i \leq n}\left(\frac{1}{T} \int_{0}^{T} S_{i t} d t\right)\right)^{+}\right] \\
= & \exp (-\gamma T) \int_{0}^{1}\left[K-\min _{1 \leq i \leq n}\left(\frac{1}{T} \int_{0}^{T} S_{i t}^{1-\alpha} d t\right)\right]^{+} d \alpha \\
= & \exp (-\gamma T) \int_{0}^{1}\left[K-\min _{1 \leq i \leq n}\left(\frac{S_{i 0}}{T} \int_{0}^{T}\right.\right. \\
& \left.\left.\exp \left(\mu_{i} t+\frac{\sqrt{3} \sigma_{i} t}{\pi} \ln \frac{1-\alpha}{\alpha}\right) d t\right)\right]^{+} d \alpha \\
= & \exp (-\gamma T) \int_{0}^{1}\left[K-\min _{1 \leq i \leq n}\left(\frac{S_{i 0}}{T} \int_{0}^{T}\right.\right. \\
& \left.\left.\exp \left(\mu_{i} t+\frac{\sqrt{3} \sigma_{i} t}{\pi} \ln \frac{\alpha}{1-\alpha}\right) d t\right)\right]^{+} d \alpha
\end{aligned}
$$

The proof is completed.

Example 9 Consider that there are $S_{10}=9, S_{20}=8, K=$ $10, T=3$ for an arithmetic Asian rainbow put on min option with two underlying stocks. In Model (3), we assume that $\gamma=0.02, \mu_{1}=-0.01, \sigma_{1}=0.04, \mu_{2}=-0.02, \sigma_{2}=0.05$. Then, $f_{2 p}^{a}=2.078$ by Theorem 15 .

Figure 9 demonstrates that the price $f_{2 p}^{a}$ is an increasing function of maturity time $T$ if other parameters are invariant.

\subsection{Geometric Asian rainbow put on min option}

Geometric Asian rainbow put on min option signifies that the holder can sell min asset at the exercise price at the time of execution. The payoff of the option relies on the geometric mean of minimum asset price within an agreed period of time.

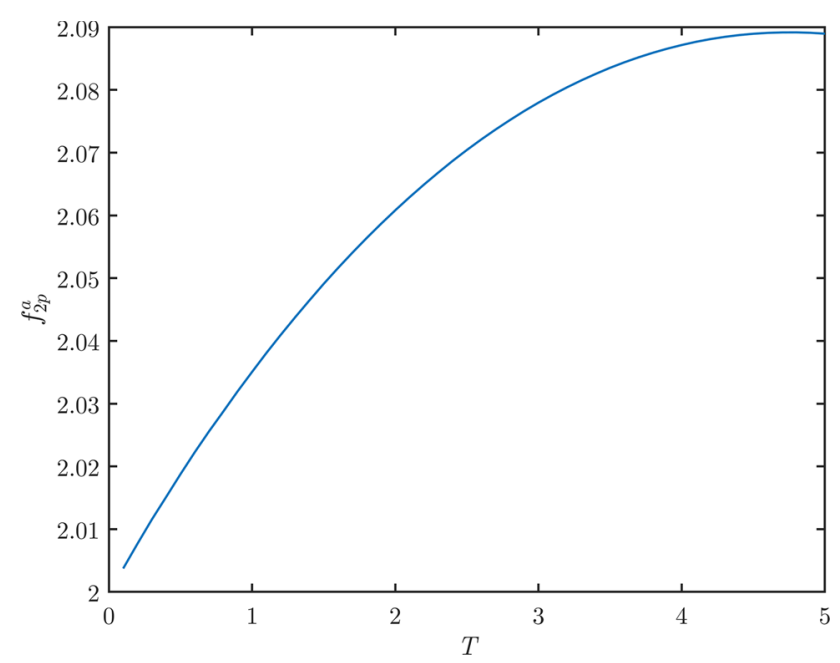

Fig. 9 Price $f_{2 p}^{a}$ concerning maturity time $T$ in Example 9 
Assume that geometric Asian rainbow put on min option possesses a maturity time $T$ and an exercise price $K$ for Model (3).

Firstly, from the holder's perspective, the payoff at time $T$ is

$$
\left[K-\min _{1 \leq i \leq n}\left(\exp \left(\frac{1}{T} \int_{0}^{T} \ln \left(S_{i t}\right) d t\right)\right)\right]^{+}
$$

Let $f_{2 p}^{g}$ be the price of geometric Asian rainbow put on min option. Thus, at the initial moment, the holder of the option owns the net return

$-f_{2 p}^{g}+\exp (-\gamma T)\left[K-\min _{1 \leq i \leq n}\left(\exp \left(\frac{1}{T} \int_{0}^{T} \ln \left(S_{i t}\right) d t\right)\right)\right]^{+}$. $T$ is

Secondly, from the seller's perspective, the payoff at time

$-\left[K-\min _{1 \leq i \leq n}\left(\exp \left(\frac{1}{T} \int_{0}^{T} \ln \left(S_{i t}\right) d t\right)\right)\right]^{+}$.

Then, at the initial moment, the net return of the seller can be expressed:

$$
f_{2 p}^{g}-\exp (-\gamma T)\left[K-\min _{1 \leq i \leq n}\left(\exp \left(\frac{1}{T} \int_{0}^{T} \ln \left(S_{i t}\right) d t\right)\right)\right]^{+}
$$

Both should own uniform expected payoff by the fair price principle, that is

$$
\begin{aligned}
& -f_{2 p}^{g}+\exp (-\gamma T) E[(K \\
& \left.\left.-\min _{1 \leq i \leq n}\left(\exp \left(\frac{1}{T} \int_{0}^{T} \ln \left(S_{i t}\right) d t\right)\right)\right)^{+}\right] \\
& =f_{2 p}^{g}-\exp (-\gamma T) E[(K \\
& \left.\left.-\min _{1 \leq i \leq n}\left(\exp \left(\frac{1}{T} \int_{0}^{T} \ln \left(S_{i t}\right) d t\right)\right)\right)^{+}\right] .
\end{aligned}
$$

From the above, we conclude that the price of geometric Asian rainbow put on min option is equal to the present value of the expected payoff.

Definition 12 Suppose that a geometric Asian rainbow put on min option for Model (3) has a maturity time $\mathrm{T}$ and an exercise price $\mathrm{K}$. Then, its price is defined by

$$
\begin{aligned}
f_{2 p}^{g} & =\exp (-\gamma T) E[(K \\
& \left.\left.-\min _{1 \leq i \leq n}\left(\exp \left(\frac{1}{T} \int_{0}^{T} \ln \left(S_{i t}\right) d t\right)\right)\right)^{+}\right] .
\end{aligned}
$$

Theorem 16 Suppose that a geometric Asian rainbow put on min option for Model (3) has a maturity time $T$ and an exercise price $K$. Then, its price is

$$
\begin{aligned}
& f_{2 p}^{g}=\exp (-\gamma T) \int_{0}^{1}\left[K-\min _{1 \leq i \leq n}\left(S_{i 0}\right.\right. \\
& \left.\left.\quad \exp \left(\frac{\mu_{i} T}{2}+\frac{\sqrt{3} \sigma_{i} T}{2 \pi} \ln \frac{\alpha}{1-\alpha}\right)\right)\right]^{+} d \alpha .
\end{aligned}
$$

Proof We show that the uncertain variable

$$
\left[K-\min _{1 \leq i \leq n}\left(\exp \left(\frac{1}{T} \int_{0}^{T} \ln \left(S_{i t}\right) d t\right)\right)\right]^{+}
$$

has an inverse uncertainty distribution

$$
\left[K-\min _{1 \leq i \leq n}\left(\exp \left(\frac{1}{T} \int_{0}^{T} \ln \left(S_{i t}^{1-\alpha}\right) d t\right)\right)\right]^{+}
$$

where

$$
S_{i t}^{1-\alpha}=S_{i 0} \exp \left(\mu_{i} t+\frac{\sqrt{3} \sigma_{i} t}{\pi} \ln \frac{1-\alpha}{\alpha}\right) .
$$

Since $\exp \left(\int_{0}^{T} \ln (x) d t / T\right)$ is an increasing function with regard to $\ln (x)$, we derive

$$
\begin{aligned}
& \left\{\left[K-\min _{1 \leq i \leq n}\left(\exp \left(\frac{1}{T} \int_{0}^{T} \ln \left(S_{i t}\right) d t\right)\right)\right]^{+}\right. \\
& \left.\quad \leq\left[K-\min _{1 \leq i \leq n}\left(\exp \left(\frac{1}{T} \int_{0}^{T} \ln \left(S_{i t}^{\alpha}\right) d t\right)\right)\right]^{+}\right\} \\
& \quad \supseteq\left\{\min _{1 \leq i \leq n}\left(\exp \left(\frac{1}{T} \int_{0}^{T} \ln \left(S_{i t}\right) d t\right)\right)\right. \\
& \left.\quad \geq \min _{1 \leq i \leq n}\left(\exp \left(\frac{1}{T} \int_{0}^{T} \ln \left(S_{i t}^{1-\alpha}\right) d t\right)\right), t \in[0, T]\right\} \\
& \quad \supseteq\left\{S_{i t} \geq S_{i t}^{1-\alpha}, t \in[0, T], 1 \leq i \leq n\right\}
\end{aligned}
$$

and

$$
\begin{aligned}
& \left\{\left[K-\min _{1 \leq i \leq n}\left(\exp \left(\frac{1}{T} \int_{0}^{T} \ln \left(S_{i t}\right) d t\right)\right)\right]^{+}\right. \\
& \left.\quad>\left[K-\min _{1 \leq i \leq n}\left(\exp \left(\frac{1}{T} \int_{0}^{T} \ln \left(S_{i t}^{1-\alpha}\right) d t\right)\right)\right]^{+}\right\} \\
& \quad \supseteq\left\{\min _{1 \leq i \leq n}\left(\exp \left(\frac{1}{T} \int_{0}^{T} \ln \left(S_{i t}\right) d t\right)\right)\right. \\
& \left.\quad<\min _{1 \leq i \leq n}\left(\exp \left(\frac{1}{T} \int_{0}^{T} \ln \left(S_{i t}^{1-\alpha}\right) d t\right)\right), t \in[0, T]\right\} \\
& \quad \supseteq\left\{S_{i t}<S_{i t}^{1-\alpha}, t \in[0, T], 1 \leq i \leq n\right\} .
\end{aligned}
$$


From Theorem 1 and Theorem 4, we have

$$
\begin{aligned}
\mathcal{M} & \left\{\left[K-\min _{1 \leq i \leq n}\left(\exp \left(\frac{1}{T} \int_{0}^{T} \ln \left(S_{i t}\right) d t\right)\right)\right]^{+}\right. \\
& \left.\leq\left[K-\min _{1 \leq i \leq n}\left(\exp \left(\frac{1}{T} \int_{0}^{T} \ln \left(S_{i t}^{1-\alpha}\right) d t\right)\right)\right]^{+}\right\} \\
& \geq \mathcal{M}\left\{S_{i t} \geq S_{i t}^{1-\alpha}, t \in[0, T], 1 \leq i \leq n\right\} \\
& =\mathcal{M}\left\{\bigcap_{i=1}^{n}\left(S_{i t} \geq S_{i t}^{1-\alpha}\right), t \in[0, T]\right\} \\
& =\bigwedge_{i=1}^{n} \mathcal{M}\left\{S_{i t} \geq S_{i t}^{1-\alpha}, t \in[0, T]\right\} \\
& =\alpha
\end{aligned}
$$

and

$$
\begin{aligned}
\mathcal{M} & \left\{\left[K-\min _{1 \leq i \leq n}\left(\exp \left(\frac{1}{T} \int_{0}^{T} \ln \left(S_{i t}\right) d t\right)\right)\right]^{+}\right. \\
> & {\left.\left[K-\min _{1 \leq i \leq n}\left(\exp \left(\frac{1}{T} \int_{0}^{T} \ln \left(S_{i t}^{1-\alpha}\right) d t\right)\right)\right]^{+}\right\} } \\
\geq & \mathcal{M}\left\{S_{i t}<S_{i t}^{1-\alpha}, t \in[0, T], 1 \leq i \leq n\right\} \\
& =\mathcal{M}\left\{\bigcap_{i=1}^{n}\left(S_{i t}<S_{i t}^{1-\alpha}\right), t \in[0, T]\right\} \\
& n \\
& \bigwedge_{i=1}^{n} \mathcal{M}\left\{S_{i t}<S_{i t}^{1-\alpha}, t \in[0, T]\right\} \\
& =1-\alpha .
\end{aligned}
$$

\section{We can obtain}

$$
\begin{aligned}
\mathcal{M} & \left\{\left[K-\min _{1 \leq i \leq n}\left(\exp \left(\frac{1}{T} \int_{0}^{T} \ln \left(S_{i t}\right) d t\right)\right)\right]^{+}\right. \\
\leq & {\left.\left[K-\min _{1 \leq i \leq n}\left(\exp \left(\frac{1}{T} \int_{0}^{T} \ln \left(S_{i t}^{1-\alpha}\right) d t\right)\right)\right]^{+}\right\} } \\
& +\mathcal{M}\left\{\left[K-\min _{1 \leq i \leq n}\left(\exp \left(\frac{1}{T} \int_{0}^{T} \ln \left(S_{i t}\right) d t\right)\right)\right]^{+}\right. \\
> & {\left.\left[K-\min _{1 \leq i \leq n}\left(\exp \left(\frac{1}{T} \int_{0}^{T} \ln \left(S_{i t}^{1-\alpha}\right) d t\right)\right)\right]^{+}\right\} } \\
= & 1
\end{aligned}
$$

by using duality axiom.
It follows from Eqs. (25)-(27) that

$$
\begin{aligned}
& \mathcal{M}\left\{\left[K-\min _{1 \leq i \leq n}\left(\exp \left(\frac{1}{T} \int_{0}^{T} \ln \left(S_{i t}\right) d t\right)\right)\right]^{+}\right. \\
& \left.\quad \leq\left[K-\min _{1 \leq i \leq n}\left(\exp \left(\frac{1}{T} \int_{0}^{T} \ln \left(S_{i t}^{1-\alpha}\right) d t\right)\right)\right]^{+}\right\}=\alpha .
\end{aligned}
$$

Thus, the uncertain variable

$$
\left[K-\min _{1 \leq i \leq n}\left(\exp \left(\frac{1}{T} \int_{0}^{T} \ln \left(S_{i t}\right) d t\right)\right)\right]^{+}
$$

has an inverse uncertainty distribution

$$
\left[K-\min _{1 \leq i \leq n}\left(\exp \left(\frac{1}{T} \int_{0}^{T} \ln \left(S_{i t}^{1-\alpha}\right) d t\right)\right)\right]^{+} .
$$

Hence, the pricing formula is

$$
\begin{aligned}
f_{2 p}^{g}= & \exp (-\gamma T) E[(K \\
& \left.\left.-\min _{1 \leq i \leq n}\left(\exp \left(\frac{1}{T} \int_{0}^{T} \ln \left(S_{i t}\right) d t\right)\right)\right)^{+}\right] \\
= & \exp (-\gamma T) \int_{0}^{1}[K \\
& \left.-\min _{1 \leq i \leq n}\left(\exp \left(\frac{1}{T} \int_{0}^{T} \ln \left(S_{i t}^{1-\alpha}\right) d t\right)\right)\right]^{+} d \alpha \\
= & \exp (-\gamma T) \int_{0}^{1}[K \\
& \left.-\min _{1 \leq i \leq n}\left(S_{i 0} \exp \left(\frac{\mu_{i} T}{2}+\frac{\sqrt{3} \sigma_{i} T}{2 \pi} \ln \frac{1-\alpha}{\alpha}\right)\right)\right]^{+} d \alpha \\
= & \exp (-\gamma T) \int_{0}^{1}[K \\
& \left.-\min _{1 \leq i \leq n}\left(S_{i 0} \exp \left(\frac{\mu_{i} T}{2}+\frac{\sqrt{3} \sigma_{i} T}{2 \pi} \ln \frac{\alpha}{1-\alpha}\right)\right)\right]^{+} d \alpha .
\end{aligned}
$$

The proof is completed.

Example 10 Consider that there are $S_{10}=9, S_{20}=8, K=$ $10, T=3$ for a geometric Asian rainbow put on min option with two underlying stocks. In Model (3), we assume that $\gamma=0.02, \mu_{1}=-0.01, \sigma_{1}=0.04, \mu_{2}=-0.02, \sigma_{2}=0.05$. Then, $f_{2 p}^{g}=2.0856$ by Theorem 16 .

Figure 10 demonstrates that the price $f_{2 p}^{g}$ is an increasing function of maturity time $T$ if other parameters are invariant.

\section{Conclusions}

By using uncertain differential equations to depict the underlying assets prices, this article explored the pricing of Asian 


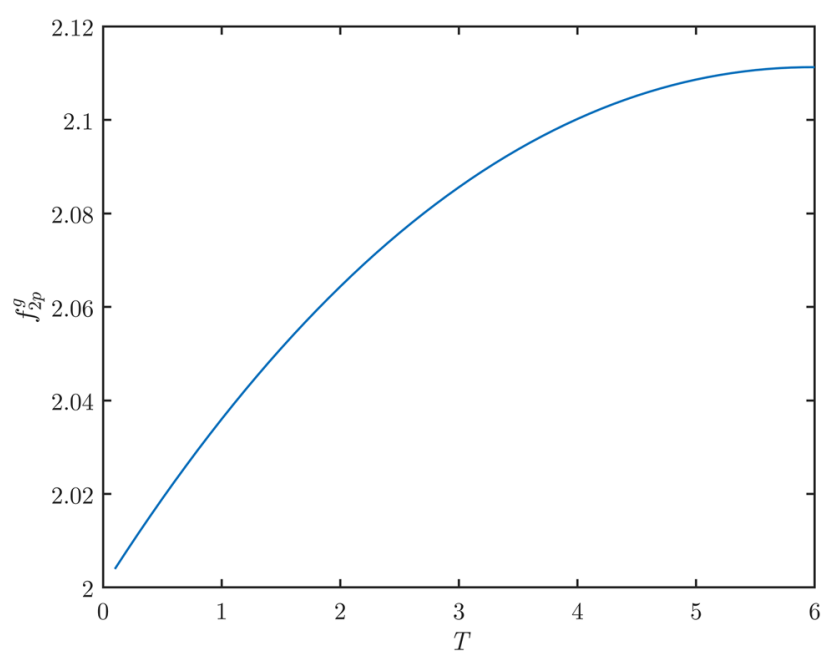

Fig. 10 Price $f_{2 p}^{g}$ concerning maturity time $T$ in Example 10

rainbow option under the uncertain stock model. We investigated three types of Asian rainbow options which are Asian put 2 and call 1 option, Asian rainbow call option and Asian rainbow put option. Furthermore, we derived the pricing formulas of corresponding option by rigorous deduction. Finally, we used some numerical examples to indicate how to utilize the option pricing formulas.

Funding This work was supported by the Natural Science Foundation of Hebei Province (No. F2020202056), Key Project of Hebei Education Department (NoZD2020125) and the Social Science Foundation of Hebei Province (No. HB18GL036).

\section{Declarations}

Conflict of interest The authors declare that we have no conflict of interest.

Ethical approval This article does not contain any studies with human participants or animals performed by any of the authors.

\section{References}

Ahmadian D, Ballestra L (2020) Pricing geometric asian rainbow options under the mixed fractional brownian motion. Phys A Stat Mech Appl 555:124458

Black F, Scholes M (1973) The pricing of options and corporate liabilities. J Polit Econ 81(3):637-654

Bin P, Fei P (2009) Pricing rainbow asian options, Systems EngineeringTheory. Practice 29(11):76-83

Cox J, Ross S (1976) The valuation of options for alternative stochastic processes. J Financ Econ 3(1-2):145-166

Corsaro S, Kyriakou I, Marazzina D, Marino Z (2019) A general framework for pricing asian options under stochastic volatility on parallel architectures. Eur. J. Oper. Res. 272(3):1082-1095
Fusai G, Kyriakou I (2016) General optimized lower and upper bounds for discrete and continuous arithmetic asian options. Math. Oper. Res. 41(2):531-559

Gao R, Liu K, Li Z, Lv R (2019) American barrier option pricing formulas for stock model in uncertain environment. IEEE Access 7:97846-97856

Ingersoll J (1987) Theory of financial decision making. Rowman \& Littlefield, New York

Johnson H (1987) Options on the maximum or the minimum of several assets. J Financ Quant Anal 22(3):277-283

Liu B (2007) Uncertainty theory, 2nd edn. Springer, Berlin

Liu B (2008) Fuzzy process, hybrid process and uncertain process. J Uncertain Syst 2(1):3-16

Liu B (2009) Some research problems in uncertainty theory. J Uncertain Syst 3(1):3-10

Liu B (2010) Uncertain risk analysis and uncertain reliability analysis. J Uncertain Syst 4(3): 163-170

Liu B (2010) Uncertainty theory: a branch of mathematics for modeling human uncertainty. Springer, Berlin

Liu B (2012) Why is there a need for uncertainty theory. J Uncertain Syst 6(1):3-10

Liu B (2013) Toward uncertain finance theory. J Uncertainty Anal Appl 1(1):Article 1

Lu Z, Yan H, Zhu Y (2019) European option pricing model based on uncertain fractional differential equation. Fuzzy Optim Decis Making 18(2):199-217

Merton R (1976) Option pricing when underlying stock returns are discontinuous. J Financ Econ 3(1-2):125-144

Margrabe W (1978) The value of an option to exchange one asset for another. J Finance 33(1):177-186

Mudzimbabwe W, Patidar K, Witbooi P (2012) A reliable numerical method to price arithmetic asian options. Appl Math Comput 218(22):10934-10942

Ouwehand P, West G (2006) Pricing rainbow options. Wilmott Maga 5:74-80

Stulz R (1982) Options on the minimum or the maximum of two risky assets: analysis and applications. J Financ Econ 10(2):161-185

Sun J, Chen X (2015) Asian option pricing formula for uncertain financial market. J Uncertainty Anal Appl 3(1):Article 11

Tian M, Yang X, Zhang Y (2019) Barrier option pricing of meanreverting stock model in uncertain environment. Math Comput Simul 166:126-143

Wu X, Zhang J (1999) Options on the minimum or the maximum of two average prices. Rev Deriv Res 3(2):183-204

Wang L, Zhang R, Yang L, Su Y, Ma F (2018) Pricing geometric asian rainbow options under fractional brownian motion. Phys A Stat Mech Appl 494:8-16

Willems S (2019) Asian option pricing with orthogonal polynomials. Quant Finance 19(4):605-618

Yao K, Chen X (2013) A numerical method for solving uncertain differential equations. J Intell Fuzzy Syst 25(3):825-832

Yao K (2013) Extreme values and integral of solution of uncertain differential equation. Journal of Uncertainty Analysis and Applications, 1(1):Article 2

Yang X, Zhang Z, Gao X (2019) Asian-barrier option pricing formulas of uncertain financial market. Chaos, Solitons Fractals 123:79-86

Zhan H, Cheng Q (2010) A simple approach to valuing asian rainbow options. Int J Electron Custom Relationsh Manag 4(1):60-76

Publisher's Note Springer Nature remains neutral with regard to jurisdictional claims in published maps and institutional affiliations. 